\title{
The Relational Syllogistic Revisited
}

\author{
IAN PRATT-HARTMANN ${ }^{1}$
}

\begin{abstract}
The relational syllogistic is an extension of the language of Classical syllogisms in which predicates are allowed to feature transitive verbs with quantified objects. It is known that the relational syllogistic does not admit a finite set of syllogism-like rules whose associated (direct) derivation relation is sound and complete. We present a modest extension of this language which does.
\end{abstract}

\section{Introduction}

By the Classical syllogistic, we mean the language featuring the four sentence-forms of standard Aristotelian syllogisms: "Every $p$ is a $q$ ", "Some $p$ is a $q$ ", "No $p$ is a $q$ " and "Some $p$ is not a $q$ ", where $p$ and $q$ are substituted by common (count) nouns. By the relational syllogistic, we mean the extension of the Classical syllogistic in which predicates are additionally allowed to feature transitive verbs with quantified objects. For example, the following is a valid argument in this language.

Some artist admires no beekeeper
Every beekeeper admires some artist
Some artist is not a beekeeper

Indeed, consider any artist, $a$, who admires no beekeeper. If he is not a beekeeper himself, the conclusion is certainly true. On the other hand, if he is a beekeeper, the second premise guarantees the existence of an artist $b$, whom he admires, and who cannot therefore be a beekeeper (since otherwise $a$ would not admire $b$ ), whence the conclusion is again true. Thus, the relational syllogistic allows us to formulate arguments that - subjectively at least - are more difficult than those we encounter

\footnotetext{
${ }^{1}$ University of Manchester
}

LiLT Volume 9

Perspectives on Semantic Representations for Textual Inference. Copyright (c) 2014, CSLI Publications. 
in the Classical syllogistic.

For the Classical syllogistic, the familiar collection of syllogisms constitutes, together with one or two ancillary rules, a (sound and) complete inference system: if an argument in this language is valid, in the sense that there is no possible world making its premises true and its conclusion false, then that validity can be demonstrated using syllogistic inference steps alone. This was first shown, in a slightly weakened form, by Corcoran Corcoran (1972) and Smiley Smiley (1973). Specifically, these authors proved certain systems of syllogism-like rules to be (sound and) refutation-complete: if an argument is valid, then an absurdity can be derived from its premises together with the negation of its conclusion. Subsequently, Pratt-Hartmann and Moss Pratt-Hartmann and Moss (2009) obtained the following results: (i) there is a finite set of syllogism-like rules in the Classical syllogistic which is sound and complete (not just refutation-complete); (ii) there is a finite set of syllogism-like rules in the relational syllogistic which is sound and refutation complete; (iii) there is no finite set of syllogism-like rules in the relational syllogistic which is sound and complete. In other words, for the relational syllogistic, indirect reasoning is essential.

This paper presents a modest extension of the relational syllogistic, which additionally features sentences of the form: "If there are $p$ 's, there are $q$ 's." The following is a valid argument in this language.

If there are artists, there are carpenters

If there are beekeepers, there are dentists

Every carpenter admires every electrician

No carpenter admires any flautist

Every dentist is an electrician

Every dentist is a flautist

No artist is a beekeeper

Indeed, suppose some artist were a beekeeper. By the first two premises, some carpenter would exist, and so would some dentist. But the remaining four premises evidently rule out the possibility that both carpenters and dentists exist, since the latter would be electricians and flautists, and hence both admired and not admired by the former.

In the sequel, we provide a finite set of syllogism-like rules for this extended relational syllogistic, and show that the resulting proof system is sound and complete. That is: the impossibility of providing a such ruleset for the relational syllogistic can be overcome by a modest increase in expressive power. Thus, the existence of a proof-system defined by a finite set of syllogism-like rules does not represent a 'boundary' with respect to the expressiveness of fragments of natural language. 
The Relational Syllogistic Revisited / 197

\section{The languages $\mathcal{S}, \mathcal{R}$ and $\mathcal{R E}$}

\subsection{Syntax and semantics}

In this section, we define the formal languages: $\mathcal{S}, \mathcal{R}$ and $\mathcal{R E}$, corresponding to the Classical syllogistic, the relational syllogistic and the extended relational syllogistic, respectively. We review known results concerning $\mathcal{S}$ and $\mathcal{R}$ and, in particular, give a sketch of the proof that $\mathcal{R}$ does not admit a finite set of syllogistic rules defining a sound and complete proof system. The motivation for defining the language $\mathcal{R E}$ as we have should at that point be obvious.

Let $\mathbf{P}$ and $\mathbf{R}$ be countably infinite sets. We call the elements of $\mathbf{P}$ unary atoms and the elements of $\mathbf{R}$ binary atoms; we use the (possibly decorated) letters $o, p, q$ to range over unary atoms, and $r$ to range over binary atoms. A unary literal is an expression of the form $p$ or $\bar{p}$, and a binary literal is an expression of the form $r$ or $\bar{r}$; we use $\ell, m$ to range over unary literals, and $t$ to range over binary literals. A unary literal is called positive if it is a unary atom, otherwise negative; similarly for binary literals. The set of $c$-terms is defined by

$$
c:=\ell \quad|\quad \forall(p, t) \quad| \quad \exists(p, t) ;
$$

we use $c, d$ to range over c-terms. The set of $\mathcal{S}$-formulas is defined by

$$
\varphi:=\forall(p, \ell) \quad \mid \quad \exists(p, \ell) ;
$$

the set of $\mathcal{R}$-formulas is defined by

$$
\varphi:=\forall(p, c) \quad \mid \quad \exists(p, c) ;
$$

and the set of $\mathcal{R E}$-formulas is defined by

$$
\varphi:=\forall(p, c) \quad|\quad \exists(p, c) \quad| \quad \exists(p, q) .
$$

We refer to formulas of the form $\exists(p, c)$ as existential formulas, and to formulas of the forms $\forall(p, c)$ and $\exists(p, q)$ as universal formulas,

Intuitively, the elements of $\mathbf{P}$ stand for common count-nouns"artist", "beekeeper", "carpenter", etc.-and those of $\mathbf{R}$ for transitive verbs-"despises", "envies", etc. We then read the c-terms $\forall(p, r)$ and $\exists(p, r)$ as "thing which $r$ 's every $p$ " and "thing which $r$ 's some $p$ ", respectively; and we read the sentence forms $\forall(p, c)$ and $\exists(p, c)$ as "Every $p$ is a $c$ " and "Some $p$ is a $c$ ", respectively. Thus we have the following informal glosses for $\mathcal{R}$-formulas.

$$
\begin{array}{ll}
\exists(\text { artist, beekeeper }) & \text { Some artist is a beekeeper } \\
\forall(\text { artist, } \forall \text { (beekeeper, admire })) & \text { Every artist (is a thing which) } \\
& \text { admires every beekeeper. }
\end{array}
$$

A negative unary literal $\bar{p}$ is then read as "is not a $p$ ", and a negative binary literal $\bar{r}$ as "does not $r$ ", but with negation having narrow scope 
with respect to the quantification of the predicate. In idiomatic English:

$$
\begin{aligned}
& \exists \text { (artist, } \overline{\text { beekeeper }}) \quad \text { Some artist is not a beekeeper } \\
& \forall(\text { artist, } \forall(\text { beekeeper, admire })) \quad \text { No artist admires any beekeeper. }
\end{aligned}
$$

Finally, the additional forms $\exists(p, q)$ in $\mathcal{R E}$ are read "If there are $p$ 's, then there are q's," thus:

$\exists$ (artist, beekeeper) If there are artists, then there are beekeepers.

We use the symbol $\mathcal{S}$ to denote the set of all $\mathcal{S}$-formulas, and similarly for $\mathcal{R}$ and $\mathcal{R E}$. When the language in question $(\mathcal{S}, \mathcal{R}$ or $\mathcal{R E})$ is clear from context, we speak simply of formulas, and we use $\varphi, \psi$ to range over formulas of that language. Notice that none of these languages includes formulas such as $\forall(\bar{p}, q)$ or $\exists(p, \forall(\bar{q}, r))$. Thus, we cannot say "Every non-artist is a beekeeper" or "Some artist admires every non-beekeeper" etc. We comment on the significance of this restriction below.

The semantics of these languages is given in the standard way. A structure is a triple $\mathfrak{A}=\left\langle A,\left\{p^{\mathfrak{A}}\right\}_{p \in \mathbf{P}},\left\{r^{\mathfrak{A}}\right\}_{r \in \mathbf{R}}\right\rangle$, where $A$ is a nonempty set, $p^{\mathfrak{A}} \subseteq A$, for every $p \in \mathbf{P}$, and $r^{\mathfrak{A}} \subseteq A \times A$, for every $r \in \mathbf{R}$. The set $A$ is called the domain of $\mathfrak{A}$. We extend the maps $p \mapsto p^{\mathfrak{A}}$ and $r \mapsto r^{\mathfrak{A}}$ to all unary and binary literals by setting, for any $p, r$ :

$$
\begin{aligned}
& \bar{p}^{\mathfrak{A}}=A \backslash p^{\mathfrak{A}} \\
& \bar{r}^{\mathfrak{A}}=(A \times A) \backslash r^{\mathfrak{A}}
\end{aligned}
$$

and thence to all c-terms by setting, for any $p, t$ :

$$
\begin{aligned}
& \forall(p, t)^{\mathfrak{A}}=\left\{a \in A \mid \text { for all } b \in p^{\mathfrak{A}},\langle a, b\rangle \in t^{\mathfrak{A}}\right\} \\
& \exists(p, t)^{\mathfrak{A}}=\left\{a \in A \mid \text { there exists } b \in p^{\mathfrak{A}} \text { such that }\langle a, b\rangle \in t^{\mathfrak{A}}\right\} .
\end{aligned}
$$

If $a \in c^{\mathfrak{A}}$, we say that a satisfies $c$ (in $\mathfrak{A}$ ), and think of $a$ as having the property denoted by $c$, according to the structure $\mathfrak{A}$. If $\ell=\bar{p}$ is a negative unary literal, we write $\bar{\ell}=p$; similarly for binary literals. We write $\bar{\forall}=\exists$ and $\bar{\exists}=\forall$. For any c-term of the form $Q(p, t)$ with $Q \in\{\forall, \exists\}$, we write $\bar{c}=\bar{Q}(p, \bar{t})$. Thus, for any c-term $c,(\bar{c})^{\mathfrak{A}}=A \backslash c^{\mathfrak{A}}$.

Having defined the notion of satisfaction for c-terms relative to a structure, we define the truth-relation $\models$ between structures and formulas as follows:

$$
\begin{aligned}
& \mathfrak{A}=\forall(p, c) \text { iff } p^{\mathfrak{A}} \subseteq c^{\mathfrak{A}} \\
& \mathfrak{A}=\exists(p, c) \text { iff } p^{\mathfrak{A}} \cap c^{\mathfrak{A}} \neq \emptyset \\
& \mathfrak{A}=\exists(p, q) \text { iff } p^{\mathfrak{A}} \neq \emptyset \text { implies } q^{\mathfrak{A}} \neq \emptyset .
\end{aligned}
$$

If $\mathfrak{A}=\theta$, we say that $\theta$ is true in $\mathfrak{A}$. We take false to mean not true. As usual, when $\Theta$ is a set of formulas, we write $\mathfrak{A} \models \Theta$ if $\mathfrak{A} \models \theta$ for 
all $\theta \in \Theta$; in this case, we say that $\mathfrak{A}$ is a model of $\Theta$, and that $\Theta$ is satisfiable. If, for every structure $\mathfrak{A}, \mathfrak{A}=\Theta$ implies $\mathfrak{A}=\theta$, then we write $\Theta \models \theta$, and we say the argument from premises $\Theta$ to conclusion $\theta$ is valid, or simply $\Theta$ entails $\theta$. A formula of the form $\exists(p, \bar{p})$ is evidently true in no structure; indeed, no other $\mathcal{R E}$-formulas have this property. We consequently refer to any formula of this form as an absurdity, and use $\perp$ to stand, indifferently, for any absurdity.

It is easy to see that the formulas $\exists(p, q)$ and $\exists(q, p)$ are true in exactly the same structures, as indeed are the formulas $\forall(p, \bar{q})$ and $\forall(q, \bar{p})$. For this reason, we henceforth regard these pairs as identical, silently transforming one into the other as required. Indeed, we allow ourselves to write any $\mathcal{R E}$-formula $\exists(p, c)$ as $\exists(c, p)$, and any $\mathcal{R E}$-formula $\forall(p, c)$ as $\forall(\bar{c}, \bar{p})$, again performing these transformations silently, as required. This (inessential) notational shortcut will allow inference rules to be stated more succinctly. It is likewise easy to see that the formula $\exists(p, c)$ is true in exactly those structures in which $\forall(p, \bar{c})$ is false (and vice versa); moreover, $\exists(p, q)$ is true in exactly those structures in which $\exists(p, p)$ and $\forall(q, \bar{q})$ are not both true. Thus, the language $\mathcal{R E}$ in effect allows negation of formulas. We remark that $\exists(p, \bar{q})$ is not an $\mathcal{R E}$ formula.

\subsection{Proof-theory}

Let $\mathcal{L}$ be any of the languages $\mathcal{S}, \mathcal{R}$ or $\mathcal{R E}$. A syllogistic rule (or simply: rule) in $\mathcal{L}$ is a pair $\Theta / \theta$, where $\Theta$ is a finite set (possibly empty) of $\mathcal{L}$ formulas, and $\theta$ an $\mathcal{L}$-formula. We generally display rules with premises and conclusion separated by a horizontal line. Thus, the rules

$$
\frac{\forall(p, q) \quad \forall(o, p)}{\forall(o, q)} \quad \frac{\forall(p, \bar{q}) \quad \forall(o, p)}{\forall(o, \bar{q})}
$$

correspond to the familiar syllogisms Barbara and Celarent:

$$
\frac{\text { Every } p \text { is a } q \text { Every } o \text { is a } p}{\text { Every } o \text { is a } q} \frac{\text { No } p \text { is a } q \text { Every } o \text { is a } p}{\text { No } o \text { is a } q} \text {. }
$$

To save space, and using the convention that $\ell$ ranges over literals, we can merge these rules as follows:

$$
\frac{\forall(p, \ell) \quad \forall(o, p)}{\forall(o, \ell)}\left(\mathrm{Al}^{\prime}\right) .
$$

We employ this convention heavily in the sequel, with $\ell$ ranging over unary literals, $t$ over binary literals, $c, d$ over c-terms, and $\psi$ over formulas. We remark that the label $\left(\mathrm{A} 1^{\prime}\right)$ is simply there to identify uses of this rule in derivations, and has no formal significance.

We are invited to think of a set of rules $\mathbf{X}$ as specifying the allowed 
steps in a derivation involving a set of formulas, $\Theta$, (the premises), and a formula, $\theta$ (the conclusion). Any derivation has the shape of a tree whose leaves are labelled with elements of $\Theta$ (repeats allowed), and whose root is labelled with $\theta$. We write $\Theta \vdash_{\mathbf{x}} \theta$ to indicate the existence of such a derivation. Thus, for example, if $X$ contains the rule $\left(\mathrm{A} 1^{\prime}\right)$, the derivation

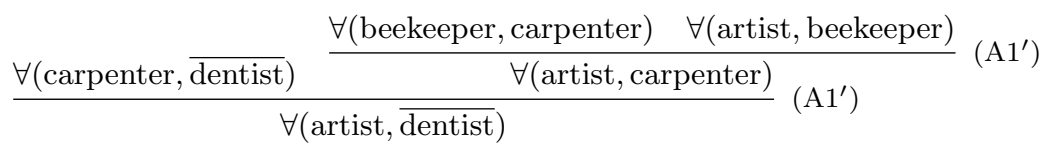

shows that

$$
\begin{aligned}
\{\forall(\text { artist }, \text { beekeeper }), & \forall \text { (beekeeper, carpenter }), \\
& \forall(\text { carpenter, } \overline{\text { dentist }})\} \vdash_{x} \forall(\text { artist, } \overline{\text { dentist }}) .
\end{aligned}
$$

It is important to realize in this regard that all proof systems considered here are direct, proceeding step-by-step from premises to conclusion. In particular, they incorporate no mechanism of indirect proof, in which a conclusion is derived by first supposing its negation and then deriving an absurdity. (We hasten to add that there is nothing wrong with indirect proofs: we are simply interested in the circumstances under which they are dispensable.)

The rule-sets $X$ of most interest to us are those whose associated derivation relation, $\vdash_{\mathrm{x}}$, constitutes necessary and sufficient conditions for entailment. Formally, we say that $\vdash_{\mathrm{x}}$ is sound if $\Theta \vdash_{\mathrm{x}} \theta$ implies $\Theta \models \theta$, and complete if $\Theta \models \theta$ implies $\Theta \vdash_{\mathbf{x}} \theta$. In this paper, we shall be concerned exclusively with finite rule-sets $X$, since there trivially exist infinite (computable) rule-sets for $\mathcal{S}, \mathcal{R}$ and $\mathcal{R} \mathcal{E}$ whose associated derivation relation is sound and complete. A set of formulas $\Theta$ is said to be inconsistent (with respect to a derivation relation $\vdash$ ) if there is a derivation of some absurdity from $\Theta$. We say that $\vdash_{x}$ is refutationcomplete if any unsatisfiable set of formulas is inconsistent. It is easy to see that completeness implies refutation-completeness, but not, it transpires, vice versa. Indeed, the following results were shown in PrattHartmann and Moss Pratt-Hartmann and Moss (2009).

Theorem 2.1. There exists a finite set $X$ of syllogistic rules in $\mathcal{S}$ such that $\vdash_{\mathrm{X}}$ is sound and complete.

Theorem 2.2. There exists a finite set $X$ of syllogistic rules in $\mathcal{R}$ such that $\vdash_{\mathrm{X}}$ is sound and refutation-complete.

Theorem 2.3. There exists no finite set $X$ of syllogistic rules in $\mathcal{R}$ such that $\vdash_{\mathrm{x}}$ is sound and complete. 
The sole result of this paper is

Theorem 2.4. There exists a finite set $X$ of syllogistic rules in $\mathcal{R E}$ such that $\vdash_{\mathrm{x}}$ is sound and complete.

We are now in a position to understand the motivation for the particular language, $\mathcal{R E}$, that we are concerned with in this paper. To do so, we need to consider the proof Theorem 2.3, which we sketch here informally; for full details, see Pratt-Hartmann and Moss Pratt-Hartmann and Moss (2009), pp. $661 \mathrm{ff}$.

Sketch proof of Theorem 2.3. For $n$ positive, let $p_{1}, \ldots, p_{n}$ be distinct unary atoms, $r$ a binary atom, $\gamma_{n}$ the $\mathcal{R}$-formula $\forall\left(p_{1}, \exists\left(p_{n}, r\right)\right)$ and $\Gamma_{n}$ the set of $\mathcal{R}$-formulas:

$$
\begin{array}{ll}
\forall\left(p_{i}, \exists\left(p_{i+1}, r\right)\right) & (1 \leq i<n) \\
\forall\left(p_{1}, \forall\left(p_{n}, r\right)\right) . &
\end{array}
$$

We claim that $\Gamma_{n} \models \gamma_{n}$. Indeed, suppose $\mathfrak{A} \models \Gamma_{n}$. If $p_{1}^{\mathfrak{A}}=\emptyset$, then trivially $\mathfrak{A}=\gamma_{n}$; on the other hand, if $p_{1}^{\mathfrak{A}} \neq \emptyset$, a simple induction using the formulas $\forall\left(p_{i}, \exists\left(p_{i+1}, r\right)\right)$ shows that $p_{n}^{\mathfrak{A}} \neq \emptyset$, whence, from $\forall\left(p_{1}, \forall\left(p_{n}, r\right)\right), \mathfrak{A} \models \gamma_{n}$. Call any formula of the form $\forall(p, p)$ trivial. For any $i(1 \leq i \leq n)$, let $\Gamma_{n, i}$ be the result from removing from $\Gamma_{n}$ the formula $\forall\left(p_{i}, \exists\left(p_{i+1}, r\right)\right)$. It can be shown, by a detailed examination of cases, that $\Gamma_{n, i}$ entails no non-trivial $\mathcal{R}$-formulas that are not already in $\Gamma_{n}$. Now consider any finite, non-empty set $X$ of rules in $\mathcal{R}$, and let $n$ be greater than the maximum number of premises in any of these rules. For any instance, $\Theta / \theta$, of one of these rules, if $\Theta \subseteq \Gamma_{n}$, then $\Theta \subseteq \Gamma_{n, i}$ for some $i(1 \leq i \leq n)$. Hence, if the rules of $\mathrm{X}$ are valid, $\theta$ must be trivial or contained in $\Gamma_{n}$. In other words, the rules of $X$ never yield non-trivial conclusions outside $\Gamma_{n}$, and in particular never yield $\gamma_{n}$. Therefore $\vdash_{\mathrm{x}}$ is not complete.

It is easy to see what is going wrong here: $\Gamma_{n, i}$ does indeed entail $\exists\left(p_{1}, p_{j}\right)$ for $1 \leq j<i$; however, these formulas are not in $\mathcal{R}$, and so cannot feature in $\mathcal{R}$-derivations. But now suppose we work within the language $\mathcal{R E}$, where we can write rules

$$
\frac{\forall(p, \exists(q, r))}{\exists(p, q)} \text { (C4) } \quad \frac{\exists(o, p) \quad \exists(p, q)}{\exists(o, q)} \text { (C2) } \frac{\forall(p, \forall(q, t)) \quad \exists(p, q)}{\forall(p, \exists(q, t))} \text { (D1). }
$$


We may derive $\exists\left(p_{1}, p_{2}\right), \exists\left(p_{1}, p_{3}\right)$ and $\exists\left(p_{1}, p_{4}\right)$ as follows:

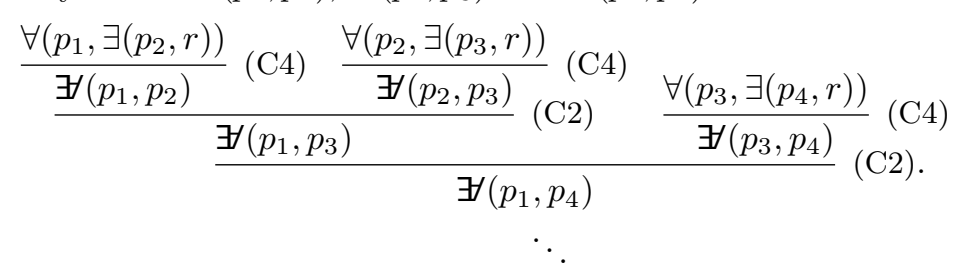

Continuing in this way, we can derive $\exists \exists\left(p_{1}, p_{i}\right)$ for all $i(2 \leq i \leq n)$, and hence derive $\gamma_{n}$, thus:

$$
\begin{aligned}
& \vdots \\
& \frac{\exists\left(p_{1}, p_{n}\right) \quad \forall\left(p_{1}, \forall\left(p_{n}, r\right)\right)}{\forall\left(p_{1}, \exists\left(p_{n}, r\right)\right)} \text { (D1) }
\end{aligned}
$$

The existence of such a derivation is no accident: formulas of the form $\exists(p, q)$ are precisely what we need to add to $\mathcal{R}$ in order to secure the existence of a sound and complete (finite) set of rules.

We mentioned above that the languages $\mathcal{S}$ and $\mathcal{R}$ do not include the formulas $\forall(\bar{p}, q)$ or $\exists(\bar{p}, \bar{q})$, and indeed that $\mathcal{R}$ does not feature the c-terms $\forall(\bar{p}, t)$ or $\exists(\bar{p}, t)$. That is, we cannot say in these languages "Every non-artist is a beekeeper", or "Every artist admires some nonbeekeeper". Extensions of $\mathcal{S}$ and $\mathcal{R}$ featuring such 'noun-level' negation were investigated in detail in Pratt-Hartmann and Moss PrattHartmann and Moss (2009), where they are denoted by $\mathcal{S}^{\dagger}$ and $\mathcal{R}^{\dagger}$, respectively. It was shown there that $\mathcal{S}^{\dagger}$ has a finite system of syllogistic rules which is sound and complete; however, there can be no such system for $\mathcal{R}^{\dagger}$, even with un-restricted use of reductio ad absurdum. It was further shown that the satisfiability problem for $\mathcal{R}^{\dagger}$ is ExPTIMEcomplete. It then follows from the fact that PTime $\neq$ ExPTime that no extension of $\mathcal{R}^{\dagger}$ can have a sound and complete (or indeed sound and refutation-complete) finite set of syllogistic rules. Therefore, noun-level negation will not be further discussed in this paper.

\subsection{A sound and complete rule-set for $\mathcal{R E}$}

We now proceed to define a rule-set, RE, which we later show to be sound and complete for $\mathcal{R E}$, under application of the proof-theoretic machinery outlined above, thus proving Theorem 2.4. Recall that we are to regard $\exists(c, p)$ as an alternative way of writing the formula $\exists(p, c)$, and similarly for the pair $\forall(\bar{c}, \bar{p}), \forall(p, c)$. These alternations will be performed silently in derivations, whenever required. Since RE is very complicated, we divide the rules into groups, with brief remarks concerning their validity. 
The first group consists of simple generalizations of familiar syllogistic rules:

$$
\begin{array}{lll}
\frac{\forall(p, q) \quad \forall(q, c)}{\forall(p, c)} \text { (A1) } & \frac{\forall(p, c) \quad \forall(q, \bar{c})}{\forall(p, \bar{q})} \text { (A2) } \frac{\exists(p, c)}{\exists(p, p)} \text { (A3) } \\
\frac{\exists(p, c) \quad \forall(p, q)}{\exists(q, c)} \text { (A4) } \frac{\exists(p, c) \quad \forall(q, \bar{c})}{\exists(p, \bar{q})} \text { (A5) } \frac{\frac{\exists(p, q) \quad \forall(q, c)}{\exists(p, c)}}{\exists(p, \bar{p})} \text { (A7) } & \frac{\forall(p, \bar{p})}{\forall(p, c)} \text { (A8) } & \overline{\forall(p, p)} \text { (A9). }
\end{array}
$$

Most of these rules should be self-explanatory: indeed, we have already met the rule (A1), though in the slightly restricted form $\left(\mathrm{A} 1^{\prime}\right)$, where $c$ was limited to literals. The rule (A7) is the rule of ex falso quodlibet, and allows us to infer anything from an absurdity. Do not confuse this rule with the strategy of reductio ad absurdum, which allows us to retract a premise and infer its negation having derived an absurdity: reductio ad absurdum cannot be understood - in the technical sense employed in this paper - as a syllogistic rule. The rule (A9) allows us to infer $\forall(p, p)$ from no premises.

The second group consists of rules governing quantified predicates. In these rules, the subject-quantifier, $Q$, stands for either $\forall$ or $\exists$.

$$
\begin{array}{ll}
\frac{Q(o, \forall(p, t)) \quad \exists(p, q)}{Q(o, \exists(q, t))} \text { (B1) } & \frac{Q(o, \forall(q, t)) \quad \forall(p, q)}{Q(o, \forall(p, t))} \\
\frac{Q(o, \exists(p, t)) \quad \forall(p, q)}{Q(o, \exists(q, t))} \text { (B3) } & \frac{\forall(q, \bar{q})}{\forall(p, \forall(q, t))} \text { (B4) } \\
\frac{\exists(p, \exists(q, t))}{\exists(q, q)} \text { (B5). } &
\end{array}
$$

These rules are almost trivially valid, and require no comment. 
204 / IAn Pratt-Hartmann

The third group consists of rules governing the quantifier $\exists$

$$
\begin{aligned}
& \overline{\exists(p, p)}(\mathrm{C} 1) \\
& \frac{\exists(o, p) \quad \exists(p, q)}{\exists(o, q)}(\mathrm{C} 2) \quad \frac{\forall(p, q)}{\exists(p, q)}(\mathrm{C} 3) \\
& \frac{\forall(p, \exists(q, r))}{\exists(p, q)}(\mathrm{C} 4) \quad \frac{\exists(q, q)}{\exists(p, q)} \text { (C5) } \quad \frac{\exists(p, p) \quad \exists(p, q)}{\exists(q, q)} \text { (C6) } \\
& \frac{\forall(q, \bar{q}) \quad \exists(p, q)}{\forall(p, c)}(\mathrm{C} 7) .
\end{aligned}
$$

Notice that $(\mathrm{C} 2)$ expresses the transitivity of the relation $\exists(p, q)$. The rule $(\mathrm{C} 7)$ may be surprising at first sight. The premises assert that there are no $q \mathrm{~s}$, and that if there are $p \mathrm{~s}$, then there are $q \mathrm{~s}$; it follows, of course, that there are no $p \mathrm{~s}$, and hence that every $p$ is a $c$, for any c-term $c$.

The fourth group concerns interactions between formulas with quantified predicates and formulas involving the quantifier $\exists$

$$
\begin{aligned}
& \frac{\forall(p, \forall(q, t)) \quad \exists(p, q)}{\forall(p, \exists(q, t))} \text { (D1) } \\
& \frac{\exists(q, q) \quad \exists(p, o) \quad \forall(o, q) \quad \forall(o, \forall(p, r))}{\exists(q, \forall(p, r))} \text { (D2) } \\
& \frac{\exists(p, p) \quad \exists(q, o) \quad \forall(o, \bar{q}) \quad \forall(o, p)}{\exists(p, \bar{q})}(\mathrm{D} 3) \\
& \frac{\forall\left(p, \forall\left(q^{\prime}, r\right)\right) \quad \forall(o, q) \quad \forall\left(o, q^{\prime}\right) \quad \exists(p, o)}{\forall(p, \exists(q, r))}(\mathrm{D} 4) \\
& \frac{\forall\left(o, \forall\left(o^{\prime}, t^{\prime}\right)\right) \quad \forall\left(o, \forall\left(o^{\prime}, \bar{t}^{\prime}\right)\right) \quad \exists(p, o) \quad \exists\left(q, o^{\prime}\right)}{\forall(p, \forall(q, t))} \text { (D5) } \\
& \frac{\forall\left(o, \forall\left(o^{\prime}, t^{\prime}\right)\right) \quad \forall\left(o, \forall\left(o^{\prime}, \bar{t}^{\prime}\right)\right) \quad \exists(p, o) \quad \exists\left(q, o^{\prime}\right)}{\forall(p, \bar{q})} \text { (D6). }
\end{aligned}
$$

Consider (D1). The premises assert that every $p$ is related by $t$ to every $q$, and that, if $p$ s exist then $q$ 's exist. If no $p$ s exist, the conclusion is 
vacuously true; otherwise, we may choose some $q$ to which every $p$ is related, and the conclusion is certainly true.

The fifth group allows slightly less obvious inferences involving formulas with quantified predicates. Rule (E2) has the form of the argument displayed on the first page of this article, and we have already demonstrated its validity; the other rules in this group may be treated similarly.

$$
\begin{aligned}
& \frac{\forall(o, \forall(p, t)) \quad \exists(o, \forall(q, \bar{t}))}{\forall(p, \bar{q})} \text { (E1) } \quad \frac{\forall(q, \exists(p, t)) \quad \exists(p, \forall(q, \bar{t}))}{\exists(p, \bar{q})} \\
& \frac{\exists(p, \exists(p, t)) \quad \forall(q, \forall(q, \bar{t}))}{\exists(p, \bar{q})} \text { (E3). }
\end{aligned}
$$

The sixth group allows us to conclude, from premises with quantified predicates, a conclusion of the form $\exists(p, \bar{q})$ :

$$
\begin{aligned}
& \frac{\exists(p, \forall(o, t)) \quad \forall(q, \forall(q, \bar{t})) \quad \forall(o, p) \quad \exists(q, o)}{\exists(p, \bar{q})}(\mathrm{F} 1) \\
& \frac{\exists(p, \forall(q, t)) \quad \forall(q, \forall(o, \bar{t})) \quad \forall(o, p) \quad \exists((q, o)}{\exists(p, \bar{q})}(\mathrm{F} 2) \\
& \frac{\forall(o, \exists(p, t)) \quad \forall(o, \forall(q, \bar{t})) \quad \exists(q, o) \quad \exists(p, p)}{\exists(p, \bar{q})} \text { (F3) } \\
& \frac{\forall\left(o, \forall\left(o^{\prime}, t\right)\right) \quad \forall(o, \forall(q, \bar{t})) \quad \exists(q, o) \quad \exists\left(q, o^{\prime}\right) \quad \forall\left(o^{\prime}, p\right) \quad \exists(p, p)}{\exists(p, \bar{q})} \text { (F4) } \\
& \frac{\forall(o, \exists(p, t)) \quad \forall(q, \forall(q, \bar{t})) \quad \exists(q, o) \quad \forall(o, p) \quad \exists(p, p)}{\exists(p, \bar{q})} \text { (F5) } \\
& \frac{\forall(o, \forall(q, t)) \quad \forall(q, \exists(p, \bar{t})) \quad \exists(q, o) \quad \forall(o, p) \quad \exists(p, p)}{\exists(p, \bar{q})}(\mathrm{F} 6) \\
& \frac{\forall\left(o^{\prime}, \forall(o, t)\right) \quad \forall(q, \forall(o, \bar{t})) \quad \exists\left(q, o^{\prime}\right) \quad \forall\left(o^{\prime}, p\right) \quad \exists(q, o) \quad \exists(p, p)}{\exists(p, \bar{q})} \\
& \frac{\forall\left(o^{\prime}, \forall(o, t)\right) \quad \forall(q, \forall(q, \bar{t})) \quad \exists\left(q, o^{\prime}\right) \quad \forall\left(o^{\prime}, p\right) \quad \exists(q, o) \quad \forall(o, p) \quad \exists(p, p)}{\exists(p, \bar{q})} \\
& \frac{\forall\left(o^{\prime}, \forall(q, t)\right) \quad \forall(q, \forall(o, \bar{t})) \quad \exists\left(q, o^{\prime}\right) \quad \forall\left(o^{\prime}, p\right) \quad \exists(q, o) \quad \forall(o, p) \quad \exists(p, p)}{\exists(p, \bar{q})}
\end{aligned}
$$


We demonstrate the validity of the most complicated of these rules, (F9). From the last premise, $\exists(p, p)$, let $a$ be some $p$. If $a$ is not a $q$, the conclusion is true. Otherwise, from the premises $\exists\left(q, o^{\prime}\right)$ and $\exists(q, o)$, let $b, b^{\prime}$ satisfy $o, o^{\prime}$, respectively. From $\forall(o, p)$, and $\forall\left(o^{\prime}, p\right), b$ and $b^{\prime}$ are both $p$ s. If either of these is not a $q$, the conclusion is true. Otherwise, from $\forall(q, \forall(o, \bar{t}))$, and $\forall\left(o^{\prime}, \forall(q, t)\right), b^{\prime}$ both is and is not related to $b$ by $t$ - contradiction. The validity of rules (F1)-(F8) may be demonstrated by similar arguments.

The seventh group allows us to conclude, from premises with quantified predicates, a conclusion of the form $\forall(p, \bar{q})$.

For any $o_{1}, o_{2}, o_{3}, o_{4} \in\{p, q\}$ :

$$
\begin{aligned}
& \frac{\forall\left(o_{1}, \forall\left(p^{\prime}, t\right)\right) \quad \forall\left(o_{2}, \forall\left(q^{\prime}, \bar{t}\right)\right) \quad \forall\left(o_{3}, p^{\prime}\right) \quad \forall\left(o_{4}, q^{\prime}\right)}{\forall(p, \bar{q})} \\
& \frac{\forall\left(o_{1}, \forall\left(q^{\prime}, t\right)\right) \quad \forall\left(o_{2}, \forall\left(q^{\prime}, \bar{t}\right)\right) \quad \exists\left(\left(o_{3}, o\right) \quad \forall\left(o, p^{\prime}\right) \quad \forall\left(o, q^{\prime}\right)\right.}{\forall(p, \bar{q})} \\
& \frac{\exists\left(o_{1}, o\right) \quad \forall\left(o, \forall\left(p^{\prime}, t\right)\right) \quad \forall\left(o, \forall\left(q^{\prime}, \bar{t}\right)\right) \quad \forall\left(o_{2}, p^{\prime}\right) \quad \forall\left(o_{3}, q^{\prime}\right)}{\forall(p, \bar{q})} \\
& \frac{\exists(p, o) \quad \exists\left(q, o^{\prime}\right) \quad \forall\left(o, \forall\left(p^{\prime}, t\right)\right) \quad \forall\left(o, \forall\left(q^{\prime}, \bar{t}\right)\right) \quad \forall\left(o^{\prime}, p^{\prime}\right) \quad \forall\left(o^{\prime}, q^{\prime}\right)}{\forall(p, \bar{q})}
\end{aligned}
$$

To demonstrate the validity of (G1), suppose, to the contrary, that some $p$ is a $q$. Choose any such element, $a$. Then, no matter how $o_{1}, \ldots, o_{4}$ are chosen from $p$ and $q$, the premises guarantee that $a$ is a $p^{\prime}$ and a $q^{\prime}$, and hence both is and is not related to itself by $t$ contradiction. The validity of rules (G2)-(G4) may be demonstrated by similar arguments.

We make no claim regarding the non-redundancy of the rule-set RE.

\section{Proof of main result}

This section is devoted entirely to the proof of Theorem 2.4. Soundness of $\vdash_{\mathrm{RE}}$ is straightforward, since, as may readily be verified, every rule in $\mathrm{RE}$ is valid. It therefore remains to show that, if $\Phi$ is a set of $\mathcal{R E}$ formulas, and $\psi$ is an $\mathcal{R E}$-formula such that $\Phi \models \psi$, then $\Phi \vdash_{\mathrm{RE}} \psi$. As RE is the only rule-set we shall be concerned with in the sequel, we henceforth write $\vdash$ in place of $\vdash_{\mathrm{RE}}$. In addition, we continue to take the letters $o, p, q$ to range over unary atoms, $\ell, m$ over unary literals, $r$ over binary atoms, $t$ over binary literals, $c, d$ over c-terms, and $\varphi, \psi$ 
over formulas, without further comment.

We provide the reader with some help in navigating the proof. We must show that, for any set of formulas $\Phi$ and any formula $\psi, \Phi \models \psi$ implies $\Phi \vdash \psi$. The proof is divided into a series of cases, corresponding to the possible forms of $\psi$, with each case covered by a specific lemma as follows.

\begin{tabular}{|r|l||r|l|}
\hline Form of $\psi$ & Lemma & Form of $\psi$ & Lemma \\
\hline$\exists(p, q)$ & 3.12 & $\exists(p, \bar{q})$ & 3.21 \\
$\exists(p, \exists(q, t))$ & 3.12 & $\exists(p, \forall(q, t))$ & 3.15 \\
$\forall(p, q)$ & 3.15 & $\forall(p, \bar{q})$ & 3.16 \\
$\forall(p, \exists(q, t))$ & 3.15 & $\forall(p, \forall(q, t))$ & 3.18 \\
$\exists(p, q)$ & 3.15 & & \\
\hline
\end{tabular}

We consider these cases roughly in order of difficulty - by far the most complicated being that of $\exists(p, \bar{q})$. For each case, we assume that $\Phi \models \psi$, and attempt to build a structure $\mathfrak{A}$ satisfying $\Phi$, possibly together with additional formulas. Two conditions then arise: either these formulas are jointly unsatisfiable, and the structure $\mathfrak{A}$ is shown to contain a defect (as we shall call it); or $\mathfrak{A}$ yields a model of $\Phi$, and hence of $\psi$. We then show that, on either condition, $\Phi \vdash \psi$.

\subsection{Two transitive closures}

Let $\Pi$ be a set of universal formulas. Define the binary relation $\stackrel{\Pi}{\longrightarrow}$ to be the smallest reflexive, transitive relation on unary atoms satisfying

$$
p \stackrel{\Pi}{\rightarrow} q \quad \text { if } \Pi \text { contains } \forall(p, q), \forall(p, \exists(q, t)) \text { or } \exists(p, q) .
$$

The key lemma regarding $\stackrel{\Pi}{\longrightarrow}$ is:

Lemma 3.1. If $p \stackrel{\Pi}{\rightarrow} q$, then $\Phi \vdash \exists(p, q)$.

Proof. Immediate given the rules

$$
\begin{array}{ll}
\frac{\exists}{\exists(p, p)}(\mathrm{C} 1) & \frac{\exists(o, p) \exists(p, q)}{\exists(o, q)}(\mathrm{C} 2) \\
\frac{\forall(p, q)}{\exists(p, q)}(\mathrm{C} 3) & \frac{\forall(p, \exists(q, r))}{\exists}(\mathrm{C} 4) .
\end{array}
$$

Let $\Pi$ be a set of universal formulas. Define the binary relation $\stackrel{\Pi}{\Longrightarrow}$ 
208 / IAn Pratt-Hartmann

to be the smallest reflexive, transitive relation on c-terms satisfying

$$
\begin{array}{cl}
p \stackrel{\Pi}{\Longrightarrow} c & \text { if } \forall(p, c) \in \Pi \\
\forall(q, t) \stackrel{\Pi}{\Longrightarrow} \forall(p, t) & \text { if } \forall(p, q) \in \Pi \\
\exists(p, t) \stackrel{\Pi}{\Longrightarrow} \exists(q, t) & \text { if } \forall(p, q) \in \Pi .
\end{array}
$$

The key lemma regarding $\stackrel{\Pi}{\Longrightarrow}$ is:

Lemma 3.2. If $p \stackrel{\Pi}{\Longrightarrow} d$, then $\Pi \vdash \forall(p, d)$.

Proof. Immediate given the rules

$$
\begin{array}{ll}
\frac{}{\forall(p, p)} \text { (A9) } & \frac{\forall(p, q) \quad \forall(q, c)}{\forall(p, c)} \text { (A1) } \\
\frac{\forall(o, \exists(p, t)) \quad \forall(p, q)}{\forall(o, \exists(q, t))} \text { (B3) } & \frac{\forall(o, \forall(q, t)) \quad \forall(p, q)}{\forall(o, \forall(p, t))} \text { (B2). }
\end{array}
$$

The following lemma will be used implicitly at various points in the sequel.

Lemma 3.3. If $c \stackrel{\Pi}{\Longrightarrow} q$, then $c$ is a unary atom. Indeed, if $p \stackrel{\Pi}{\Longrightarrow} q$, then: (i) $p \stackrel{\Pi}{\longrightarrow} q$; (ii) $\forall(q, t) \stackrel{\Pi}{\Longrightarrow} \forall(p, t)$; and (iii) $\exists(p, t) \stackrel{\Pi}{\Longrightarrow} \exists(q, t)$.

Proof. Obvious.

Similarly, we have:

Lemma 3.4. If $o \stackrel{\Pi}{\Longrightarrow} \exists(p, t)$ then $o \stackrel{\Pi}{\longrightarrow} p$. If $c \stackrel{\Pi}{\Longrightarrow} \exists(p, t)$ and $c$ is not a unary atom, then $c=\exists(o, t)$ for some $o$ such that $o \stackrel{\Pi}{\Longrightarrow} p$, whence $o \stackrel{\Pi}{\rightarrow} p$.

\subsection{Some technical machinery}

Let $s$ be any set of c-terms. We think of $s$ as a description of an individual: that individual satisfies all the c-terms in $s$. For any set $\Sigma$ of existential formulas, define

$$
U_{\Sigma}=\{\{p, c\} \mid \exists(p, c) \in \Sigma\}
$$

We think of $U_{\Sigma}$ as a collection of (descriptions of) individuals that obviously exist on the assumption that the formulas in $\Sigma$ are true. In addition, for any set $\Sigma$ of existential formulas and any set $\Pi$ of universal 
formulas, define

$$
\begin{aligned}
O_{\Sigma, \Pi}= & \left\{o \mid p \stackrel{\Pi}{\rightarrow} o \text { for some } p \in \bigcup U_{\Sigma}\right\} \\
& \bigcup\left\{o \mid p \stackrel{\Pi}{\rightarrow} o \text { for some } \exists(p, t) \in \bigcup U_{\Sigma}\right\} \\
V_{\Sigma, \Pi}= & U_{\Sigma} \cup\left\{\{o\} \mid o \in O_{\Sigma, \Pi}\right\} .
\end{aligned}
$$

Think of $O_{\Sigma, \Pi}$ as the set of unary predicates that must be instantiated on the assumption that the formulas in $\Sigma \cup \Pi$ are true; and think of $V_{\Sigma, \Pi}$ as a set of (descriptions of) individuals that exist on the assumption that the formulas in $\Sigma \cup \Pi$ are true.

For any set $s$ of c-terms and any set $\Pi$ of universal formulas, define

$$
s^{\Pi}=\{d \mid c \stackrel{\Pi}{\Longrightarrow} d \text { for some } c \in s\} .
$$

We think of $s^{\Pi}$ as an elaboration of the description $s$ : any individual described by $s$ is in fact described by $s^{\Pi}$, provided the formulas in $\Pi$ are all true. We remark in passing that it is useful to imagine $s^{\Pi}$ as the union of a sequence $s_{0}, s_{1}, \ldots$ of sets of c-terms, defined inductively as follows

$$
\begin{aligned}
s_{0} & =s \\
s_{3 k+1} & =s_{3 k} \cup\left\{c \mid p \in s_{3 k} \text { and } \forall(p, c) \in \Pi\right\} \\
s_{3 k+2} & =s_{3 k+1} \cup\left\{\forall(p, t) \mid \forall(q, t) \in s_{3 k+1} \text { and } \forall(p, q) \in \Pi\right\} \\
s_{3(k+1)} & =s_{3 k+2} \cup\left\{\exists(q, t) \mid \exists(p, t) \in s_{3 k+2} \text { and } \forall(p, q) \in \Pi\right\} .
\end{aligned}
$$

We refer to the sequence $s_{0}, s_{1}, \ldots$ as the staged construction of $s^{\Pi}$.

Finally, define

$$
W_{\Sigma, \Pi}=\left\{s^{\Pi} \mid s \in V_{\Sigma, \Pi}\right\} .
$$

We think of $W_{\Sigma, \Pi}$ as a collection of elaborated descriptions of the individuals that must exist on the assumption that the formulas in $\Sigma \cup \Pi$ are true. Sometimes, things can go wrong with $W_{\Sigma, \Pi}$. Suppose $w \in W_{\Sigma, \Pi}$ and, for some c-term $c$, both $c$ and $\bar{c}$ are elements of $w$ : in that case, the description $w$ cannot be satisfied, and we say that $W_{\Sigma, \Pi}$ contains a local defect at $w$. Or suppose that $u, v \in W_{\Sigma, \Pi}$ and, for some unary atoms $p, q$ and binary atom $r, u$ contains both $\forall(p, r)$ and $\forall(q, \bar{r})$, while $v$ contains both $p$ and $q$ : in that case, the descriptions $u$ and $v$ cannot be simultaneously satisfied, and we say that $W_{\Sigma, \Pi}$ contains a global defect involving $u$ and $v$. A defect is a local or global defect.

Lemma 3.5. If $\exists(p, t) \in u \in W_{\Sigma, \Pi}$, then $\{p\}^{\Pi} \in W_{\Sigma, \Pi}$.

Proof. Lemma 3.4. 
Given a set $W_{\Sigma, \Pi}$, let $A=W_{\Sigma, \Pi} \times\{0,1\}$, and define structures $\mathfrak{A}$ and $\overline{\mathfrak{A}}$ over $A$ by setting, for all $p \in \mathbf{P}$ and $r \in \mathbf{R}$ :

$$
\begin{aligned}
p^{\mathfrak{A}}=p^{\overline{\mathfrak{A}}}= & \{(u, i) \in A \mid p \in u\} \\
r^{\mathfrak{A}}= & \left\{\left\langle(u, i),\left(\{p\}^{\Pi}, 1\right)\right\rangle \in A \times A \mid \exists(p, r) \in u\right\} \cup \\
& \{\langle(u, i),(v, j)\rangle \in A \times A \mid \text { there exists } p \in v \text { s.t. } \forall(p, r) \in u\} \\
r^{\overline{\mathfrak{A}}}= & (A \times A) \backslash \\
& \left(\left\{\left\langle(u, i),\left(\{p\}^{\Pi}, 1\right)\right\rangle \in A \times A \mid \exists(p, \bar{r}) \in u\right\} \cup\right. \\
& \{\langle(u, i),(v, j)\rangle \in A \times A \mid \text { there exists } p \in v \text { s.t. } \forall(p, \bar{r}) \in u\})
\end{aligned}
$$

In the definitions of $r^{\mathfrak{A}}$ and $r^{\overline{\mathfrak{A}}}$, Lemma 3.5 ensures that we never have to worry that $\left(\{p\}^{\Pi}, 1\right)$ might not be an element of $A$. We write $\mathfrak{A}_{\Sigma, \Pi}=\mathfrak{A}$ and $\overline{\mathfrak{A}}_{\Sigma, \Pi}=\overline{\mathfrak{A}}$ when we need to make the parameters $\Sigma$ and $\Pi$ explicit.

A word of motivation is in order here. For any description $w \in W_{\Sigma, \Pi}$, the domain $A$ contains a pair of objects: $(w, 0)$ and $(w, 1)$. It helps to think of these objects as individuals which 'want' to satisfy the description $w$; the construction of $\mathfrak{A}$ and $\overline{\mathfrak{A}}$ will ensure that, in the absence of defects, these desires are satisfied. The doubling of individuals is motivated by the need to provide witnesses for certain c-terms: in $\mathfrak{A}$, the individual $\left(\{q\}^{\Pi}, 1\right)$ serves as witnesses for c-terms of the form $\exists(q, r)$, while the individual $\left(\{q\}^{\Pi}, 0\right)$ serves as a witness for c-terms of the form $\exists(q, \bar{r})$; in $\overline{\mathfrak{A}}$, these roles are reversed. Both structures interpret unary atoms in the natural way: $(w, i)$ is taken to satisfy $p$ just in case $p \in w$. For binary atoms, $\mathfrak{A}$ and $\overline{\mathfrak{A}}$ employ opposite strategies, with $\mathfrak{A}$, roughly speaking, making extensions as small as possible, and $\overline{\mathfrak{A}}$ making them as large as possible. In particular, for elements $a=(u, i)$ and $b=(v, j)$, the structure $\mathfrak{A}$ takes $a$ to stand in relation $r$ to $b$ if and only if, for some unary atom $q$, either $\exists(q, r) \in u$ and $b=\left(\{q\}^{\Pi}, 1\right)$ or $\forall(q, r) \in u$ and $q \in v$. By contrast, the structure $\overline{\mathfrak{A}}$ takes $a$ always to stand in relation $r$ to $b$ unless, for some unary atom $q$, either $\exists(q, \bar{r}) \in u$ and $b=\left(\{q\}^{\Pi}, 1\right)$ or $\forall(q, \bar{r}) \in u$ and $q \in v$. Equivalently, $\overline{\mathfrak{A}}$ takes $a$ to stand in relation $r$ to $b$ if and only if, for every unary atom $q, \exists(q, \bar{r}) \in u$ implies $b \neq\left(\{q\}^{\Pi}, 1\right)$ and $\forall(q, \bar{r}) \in u$ implies $q \notin v$.

Lemma 3.6. Let $\Sigma$ be any set of existential formulas and $\Pi$ any set of universal formulas. Let $c \in u \in W_{\Sigma, \Pi}$ and $i \in\{0,1\}$. If $W_{\Sigma, \Pi}$ contains no defects, then $a=(u, i)$ satisfies $c$ in both $\mathfrak{A}_{\Sigma, \Pi}$ and $\overline{\mathfrak{A}}_{\Sigma, \Pi}$.

Proof. Write $\mathfrak{A}=\mathfrak{A}_{\Sigma, \Pi}$ and $\overline{\mathfrak{A}}=\overline{\mathfrak{A}}_{\Sigma, \Pi}$. We show that $a \in c^{\mathfrak{A}}$. If $c$ is of 
any of the forms $p, \exists(p, r), \forall(p, r)$, this is immediate by the construction of $\mathfrak{A}$. If $c=\bar{p}$, then $p \notin u$, since $W$ contains no local defects, whence $a \in c^{\mathfrak{A}}$. If $c=\exists(p, \bar{r})$, then, $c \in u \in W_{\Sigma, \Pi}$ implies $\{p\}^{\Pi} \in W_{\Sigma, \Pi}$, and hence $b=\left(\{p\}^{\Pi}, 0\right) \in p^{\mathfrak{A}}$. To establish $a \in c^{\mathfrak{A}}$, it suffices to show that $\langle a, b\rangle \notin r^{\mathfrak{A}}$. By definition of $\mathfrak{A},\langle a, b\rangle \in r^{\mathfrak{A}}$ only if there exists $q \in\{p\}^{\Pi}$ such that $\forall(q, r) \in u$. But then, since $u$ is closed under $\stackrel{\Pi}{\Longrightarrow}$, we have $\forall(p, r) \in u$, contrary to the hypothesis that $W_{\Sigma, \Pi}$ contains no local defect. If $c=\forall(p, \bar{r})$, suppose $b=(v, j) \in p^{\mathfrak{A}}$, so that $p \in v$. To establish $a \in c^{\mathfrak{A}}$, it suffices to show that $\langle a, b\rangle \notin r^{\mathfrak{A}}$. By definition of $\mathfrak{A}$, $\langle a, b\rangle \in r^{\mathfrak{A}}$ only if, for some $q$, either (i) $v=\{q\}^{\Pi}, j=1$ and $\exists(q, r) \in u$, or (ii) $q \in v$ and $\forall(q, r) \in u$. In case (i), since $u$ is closed under $\stackrel{\Pi}{\Longrightarrow}$, we have $\exists(p, r) \in u$, contrary to the hypothesis that $W_{\Sigma, \Pi}$ contains no local defect. Case (ii) is instantly contrary to the hypothesis that $W_{\Sigma, \Pi}$ contains no global defect.

To show that $a \in c^{\overline{\mathfrak{A}}}$, we proceed in exactly the same way, except that references to $r$ and $\bar{r}$ are exchanged.

Lemma 3.7. Let $\Sigma$ be any set of existential formulas and $\Pi$ any set of universal formulas. If $W_{\Sigma, \Pi}$ contains no defects, then both $\mathfrak{A}_{\Sigma, \Pi}$ and $\overline{\mathfrak{A}}_{\Sigma, \Pi}$ are models of $\Sigma \cup \Pi$.

Proof. Write $\mathfrak{A}$ ambiguously for $\mathfrak{A}_{\Sigma, \Pi}$ or $\overline{\mathfrak{A}}_{\Sigma, \Pi}$, and $A$ for the domain of $\mathfrak{A}$. Consider first any $\varphi=\exists(p, c) \in \Sigma$. Then $a=\left(\{p, c\}^{\Pi}, 1\right) \in A$. By Lemma 3.6, $a \in p^{\mathfrak{A}} \cap c^{\mathfrak{A}}$; therefore, $\mathfrak{A} \models \varphi$. Consider next any $\varphi=\forall(p, c) \in \Pi$, and suppose $a=(u, i) \in p^{\mathfrak{A}}$. By definition of $\mathfrak{A}, p \in u$. Taking account of the fact that $u$ is closed under the relation $\stackrel{\Pi}{\Longrightarrow}$, we have $c \in u$, whence, by Lemma 3.6, $a \in c^{\mathfrak{A}}$; therefore, $\mathfrak{A} \models \varphi$. Consider finally any $\varphi=\exists(p, q) \in \Pi$, and suppose $a=(u, i) \in p^{\mathfrak{A}}$. By definition of $\mathfrak{A}, p \in u$, whence $q \in O_{\Sigma, \Pi}$, whence $b=\left(\{q\}^{\Pi}, 1\right) \in A$. By definition of $\mathfrak{A}, b \in q^{\mathfrak{A}}$; therefore, $\mathfrak{A} \models \varphi$.

\subsection{Completeness proof}

Fix a set of formulas $\Phi$ and a formula $\psi$. Our task is to demonstrate that, if $\Phi \models \psi$, then $\Phi \vdash \psi$. We consider the various forms of $\psi$ separately. Write $\Phi=\Lambda \cup \Gamma$, where $\Lambda$ is the set of universal formulas in $\Phi$, and $\Gamma$, the set of existential formulas in $\Phi$. (Recall that formulas of the form $\exists(p, q)$ count as universal.)

Lemma 3.8. If $p \in w \in W_{\Lambda, \Gamma}$, then $\Phi \vdash \exists(p, p)$.

Proof. We first prove the result for $p \in s \in V_{\Lambda, \Gamma}$. If $s=\{p, c\} \in U_{\Lambda}$, the result is immediate from the definition of $U_{\Lambda}$ and the rule (A3). So 
we may assume $s=\{p\}$, and either there exists $\left\{p^{\prime}, c^{\prime}\right\} \in U_{\Lambda}$ such that $p^{\prime} \stackrel{\Pi}{\rightarrow} p$, or there exists $\left\{p^{\prime}, \exists\left(q^{\prime}, t\right)\right\} \in U_{\Lambda}$ such that $q^{\prime} \stackrel{\Pi}{\longrightarrow} p$. Noting Lemma 3.1, we have either of the derivations

$$
\frac{\frac{\exists\left(p^{\prime}, c^{\prime}\right)}{\exists\left(p^{\prime}, p^{\prime}\right)}(\mathrm{A} 3) \quad \begin{array}{c}
\vdots \\
\exists\left(p^{\prime}, p\right)
\end{array}}{\exists(p, p)}(\mathrm{C} 6) \quad \frac{\exists\left(p^{\prime}, \exists\left(q^{\prime}, t\right)\right)}{\frac{\exists\left(q^{\prime}, q^{\prime}\right)}{(\mathrm{B} 5)}} \begin{gathered}
\exists \\
\exists\left(q^{\prime}, p\right)
\end{gathered} \text { (C6). }
$$

Finally, suppose $p^{\prime} \stackrel{\Pi}{\Longrightarrow} p$ for some $p^{\prime} \in s \in V_{\Lambda, \Gamma}$. By Lemma 3.2, $\Phi \vdash \forall\left(p^{\prime}, p\right)$, whence we have the derivation

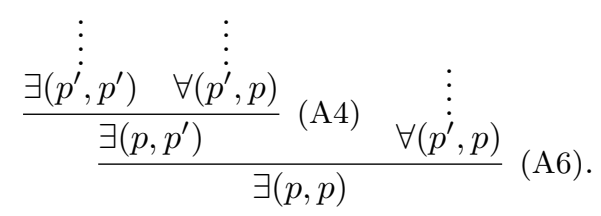

Let us say that c-terms $c$ and $d$ are sisters if they are identical, or if there exist $Q \in\{\forall, \exists\}$, unary literals $p$ and $q$, and binary literal $t$, such that $c=Q(p, t)$ and $d=Q(q, t)$. Notice in particular that no pair of c-terms of the form $c, \bar{c}$ can be sisters.

Lemma 3.9. Let $u$ be an element of $W_{\Lambda, \Gamma}$.

(i) If $c, d \in u$ with $c$ and $d$ not sisters, then there exist $o \in u$ and $Q \in\{\forall, \exists\}$ such that $\Phi \vdash Q(o, c)$ and $\Phi \vdash \bar{Q}(o, d)$.

(ii) If $q, d \in u$, then $\Phi \vdash \exists(q, d)$.

Proof. Any element of $W_{\Lambda, \Gamma}$ has the form $s^{\Gamma}$, with $s=\{p, c\} \in V_{\Lambda, \Gamma}$; recall that we earlier described the staged construction of $s^{\Gamma}$. Suppose $c$ is introduced at stage $i$ and $d$ at stage $j$ : we proceed by induction on $i+j$.

If $i=j=0$ then, $c, d \in s \in V_{\Lambda, \Gamma}$. Since $c$ and $d$ are not identical, we have $\{c, d\} \in U_{\Lambda}$, and indeed, by exchanging $c$ and $d$ if necessary $c=p$ with $\exists(p, d) \in \Phi$. From rule (A9), we have $\Phi \vdash \forall(p, p)$, so that putting $o=p$ secures the lemma. Suppose, then $i+j>0$, and assume, by exchanging $c$ and $d$ if necessary, that $i>0$. If $i=3 k+1$, let $p \in s_{3 k}$ such that $\forall(p, c) \in \Gamma$. If $p=d$, then, by Lemma 3.8, $\Phi \vdash \exists(d, d)$, so that putting $o=p$ again secures the lemma. On the other hand, if $p \neq d$, then, since $p$ is not the sister anything but itself, we have by inductive hypothesis $o$ and $Q$ such that $\Phi \vdash Q(o, p)$ and $\Phi \vdash \bar{Q}(o, d)$. But then 
we have either of the derivations

$$
\begin{array}{cc}
\vdots & \vdots \\
\frac{\forall(o, p)}{\forall(o, c)} \quad \forall(p, c) & \vdots \\
\text { (A1) } & \frac{\exists(o, p)}{\exists(o, c)} \quad \forall(p, c)
\end{array}
$$

so that $\Phi \vdash Q(o, c)$, as required.

If $i=3 k+2$, let $c=\forall(p, t)$ and suppose $\forall(q, t) \in s_{3 k+1}$ with $\forall(p, q) \in$ $\Gamma$. If $c$ is not $d$ 's sister, then neither is $\forall(q, t)$, so, by inductive hypothesis, let $o$ and $Q$ be such that $\Phi \vdash Q(o, \forall(q, t))$ and $\Phi \vdash \bar{Q}(o, d)$. But then we have the derivation

$$
\frac{Q(o, \forall(q, t)) \quad \forall(p, q)}{Q(o, \forall(p, t))}(\mathrm{B} 2) .
$$

If $i=3(k+1)$, we proceed similarly, but using rule (B3) instead of (B2).

The second statement of the lemma follows from the first by a single application of either (A4) or (A6).

Lemma 3.10. If $W_{\Lambda, \Gamma}$ contains a local defect, then $\Phi$ is inconsistent.

Proof. Let $u \in W_{\Lambda, \Gamma}$ contain $c, \bar{c}$. By Lemma 3.9 (i), there exists $o \in u$ and $Q \in\{\forall, \exists\}$ such that $\Phi \vdash Q(o, c)$ and $\Phi \vdash \bar{Q}(o, \bar{c})$. Thus, we have the derivation

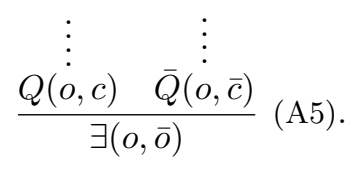

Lemma 3.11. If $W_{\Lambda, \Gamma}$ contains a global defect, then $\Phi$ is inconsistent.

Proof. Suppose $\forall(p, r), \forall(q, \bar{r}) \in u \in W_{\Lambda, \Gamma}$ and $p, q \in v \in W_{\Lambda, \Gamma}$. By Lemma 3.9 (i), there exists $o \in u$ and $Q \in\{\forall, \exists\}$ such that $\Phi \vdash$ $Q(o, \forall(p, r))$ and $\Phi \vdash \bar{Q}(o, \forall(q, \bar{t}))$. And by Lemma 3.9 (ii), $\Phi \vdash \exists(p, q)$. Thus, we have the derivation

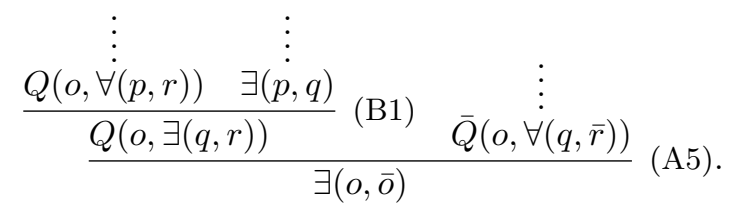


Lemma 3.12. Suppose $\Phi \models \psi$, where $\psi$ is of either of the forms $\exists(p, q)$ or $\exists(p, \exists(q, t))$. Then $\Phi \vdash \psi$.

Proof. If $W_{\Lambda, \Gamma}$ contains any local or global defects, then $\Phi$ is inconsistent, whence $\Phi \vdash \psi$ by rule (A7). Otherwise, let $\mathfrak{A}=\mathfrak{A}_{\Lambda, \Gamma}$, so that by Lemma 3.7, $\mathfrak{A} \models \Phi$, whence $\mathfrak{A} \models \psi$. If $\psi=\exists(p, q)$, then, by definition of $\mathfrak{A}$, there exists $u \in W_{\Lambda, \Gamma}$ such that $p, q, \in u$. By Lemma 3.9 (ii), $\Phi \vdash \exists(p, q)$. If $\psi=\exists(p, \exists(q, t))$, let us assume first of all that $t$ is positive, and write $t=r$. Then there exists $a=(u, i)$ and $b=(v, j)$ in $W_{\Lambda, \Gamma}$ such that $a \in p^{\mathfrak{A}}, b \in q^{\mathfrak{A}}$ and $\langle a, b\rangle \in r^{\mathfrak{A}}$. By definition of $\mathfrak{A}$, $p \in u$ and $q \in v$. Furthermore, either (a) $\exists(o, r) \in u$ and $v=\{o\}^{*}$, or (b) $\forall(o, r) \in u$ and $o \in v$. In case (a), $\Phi \vdash \forall(o, q)$ by Lemma 3.2, and $\Phi \vdash \exists(p, \exists(o, r))$ by Lemma 3.9 (ii), whence we have the derivation

$$
\begin{array}{cc}
\vdots & \vdots \\
\frac{\exists(p, \exists(o, r))}{\exists(p, \exists(q, r))} & \forall(o, q)
\end{array}
$$

In case (b), by Lemma 3.9 (ii), $\Phi \vdash \exists(o, q)$, and $\Phi \vdash \exists(p, \forall(o, r))$, whence we have the derivation

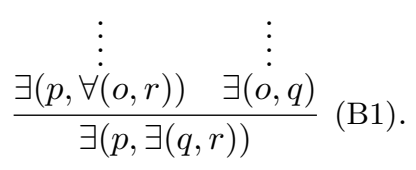

Finally, if $t=\bar{r}$ is negative, we proceed in exactly the same way, but use $\overline{\mathfrak{A}}$ in place of $\mathfrak{A}$.

We next deal with conclusions of the forms $\forall(p, q), \forall(p, \exists(q, t))$, $\exists(q, \forall(p, t)) \exists(p, q), \forall(p, \bar{q})$, and $\forall(p, \forall(q, t))$. We note in connection with the machinery introduced in Sec. 3.2 that, if $\Sigma$ and $\Sigma^{\prime}$ are sets of existential formulas and $\Gamma$ a set of universal formulas, then $W_{\Sigma \cup \Sigma^{\prime}, \Gamma}=$ $W_{\Sigma, \Gamma} \cup W_{\Sigma^{\prime}, \Gamma}$.

Lemma 3.13. If $W_{\Lambda \cup\{\exists(p, q)\}, \Gamma}$ contains a local defect, then $\Phi \vdash \forall(p, \bar{q})$.

Proof. Observe that the statement of the lemma would be unaffected

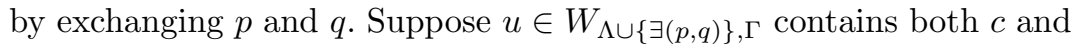
$\bar{c}$. If $u \in W_{\Lambda, \Gamma}$, then the result is secured by Lemma 3.10 and rule

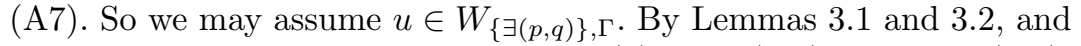
exchanging $p$ and $q$ if necessary, either (a) $\Gamma \vdash \forall(p, c)$ and $\Gamma \vdash \forall(q, \bar{c})$, or (b) there exists $o$ (possibly, $o=p$ ) such that $\Gamma \vdash \exists(p, o), \Gamma \vdash \forall(o, c)$ 
The Relational Syllogistic Revisited / 215

and $\Gamma \vdash \forall(o, \bar{c})$. Hence, we have either of the derivations

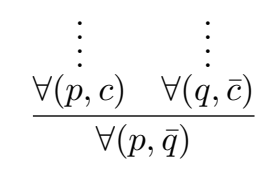

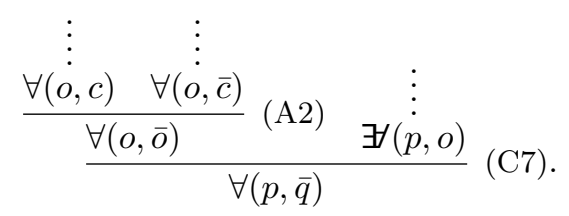

Lemma 3.14. If $W_{\Lambda \cup\{\exists(p, q)\}, \Gamma}$ contains a global defect, then $\Phi \vdash$ $\forall(p, \bar{q})$.

Proof. Observe that the statement of the lemma would be unaffected by exchanging $p$ and $q$. Suppose $u \in W_{\Lambda \cup\{\exists(p, q)\}, \Gamma}$ contains both $\forall\left(p^{\prime}, t\right)$ and $\forall\left(q^{\prime}, \bar{t}\right)$, and $v \in W_{\Lambda \cup\{\exists(p, q)\}, \Gamma}$ contains both $p^{\prime}$ and $q^{\prime}$. If $u, v \in$ $W_{\Lambda, \Gamma}$, the result is secured by Lemma 3.11 and rule (A7). So we may

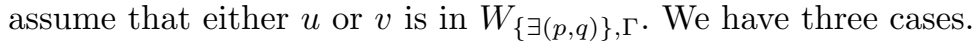

(i) Suppose first that $u \in W_{\{\exists(p, q)\}, \Gamma}$ and $v \in W_{\Lambda, \Gamma}$. By Lemmas 3.1 and 3.2, and exchanging $p$ and $q$ if necessary, either (a) $\Gamma \vdash \forall\left(p, \forall\left(p^{\prime}, t\right)\right)$ and $\Gamma \vdash \forall\left(q, \forall\left(q^{\prime}, \bar{t}\right)\right)$, or (b) there exists $o$ (possibly, $o=p$ ) such that $\Gamma \vdash \exists(p, o), \Gamma \vdash \forall\left(o, \forall\left(p^{\prime}, t\right)\right)$ and $\Gamma \vdash \forall\left(o, \forall\left(q^{\prime}, \bar{t}\right)\right)$. Furthermore, by Lemma 3.9 (ii), $\Phi \vdash \exists\left(p^{\prime}, q^{\prime}\right)$. In case (a), we have the derivation

$$
\begin{array}{ccc}
\vdots & \vdots & \vdots \\
\frac{\forall\left(p, \forall\left(p^{\prime}, t\right)\right)}{\forall\left(q, \forall\left(q^{\prime}, \bar{t}\right)\right)} \quad \exists\left(p^{\prime}, q^{\prime}\right) & \forall\left(q, \exists\left(p^{\prime}, \bar{t}\right)\right) \\
\text { (B2) }) .
\end{array}
$$

In case (b), we have the derivation

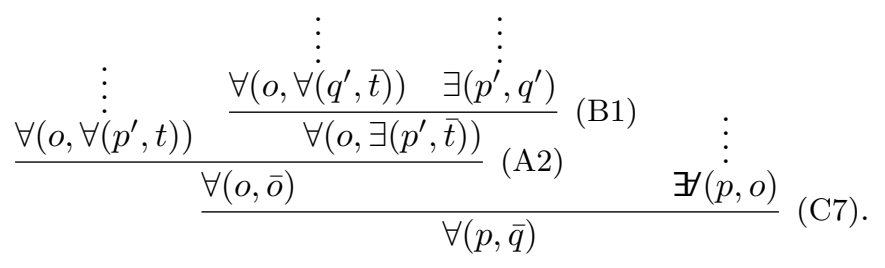

(ii) Suppose next that $u \in W_{\Lambda, \Gamma}$ and $v \in W_{\{\exists(p, q)\}, \Gamma \text {. Since } \forall\left(p^{\prime}, t\right) \text { and }}$ $\forall\left(q^{\prime}, \bar{t}\right)$ are not sisters, by Lemma 3.9 (i), there exist $o, Q$ such that $\Phi \vdash Q\left(o, \forall\left(p^{\prime}, t\right)\right)$ and $\Phi \vdash \bar{Q}\left(o, \forall\left(q^{\prime}, \bar{t}\right)\right)$. By Lemmas 3.1 and 3.2, and exchanging $p$ and $q$ if necessary, either (a) $\Gamma \vdash \forall\left(p, p^{\prime}\right)$ and $\Gamma \vdash \forall\left(q, q^{\prime}\right)$, or (b) there exists $o^{\prime}$ (possibly, that $o^{\prime}=p$ ) such that $\Gamma \vdash \exists\left(p, o^{\prime}\right)$, 
216 / IAn Pratt-Hartmann

$\Gamma \vdash \forall\left(o^{\prime}, p^{\prime}\right)$ and $\Gamma \vdash \forall\left(o^{\prime}, q^{\prime}\right)$. In case (a), we have the derivation

$$
\frac{\forall\left(q, q^{\prime}\right)}{\frac{Q\left(o, \forall\left(p^{\prime}, t\right)\right)}{\bar{Q}\left(o, \forall\left(q^{\prime}, \bar{t}\right)\right)}} \begin{gathered}
\vdots \\
\text { (E1) } \begin{array}{c}
\vdots \\
\forall\left(p, p^{\prime}\right)
\end{array}
\end{gathered}
$$

In case (b), we have the derivation

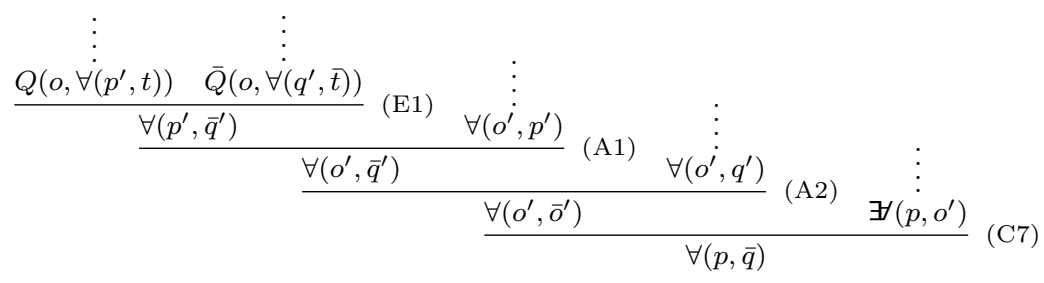

(iii) Suppose, finally, that $u, v \in W_{\{\exists(p, q)\}, \Gamma \text {. Here we have four sub- }}$ cases to consider.

(a) If $u=v=\{p, q\}^{\Gamma}$, then by Lemma 3.2, there exist $o_{1}, o_{2}, o_{3}, o_{4} \in$ $\{p, q\}$ such that $\Gamma \vdash \forall\left(o_{1}, \forall\left(p^{\prime}, t\right)\right), \Gamma \vdash \forall\left(o_{2}, \forall\left(q^{\prime}, \bar{t}\right)\right), \Gamma \vdash \forall\left(o_{3}, p^{\prime}\right)$, and $\Gamma \vdash \forall\left(o_{4}, q^{\prime}\right)$. Thus we have the derivation

$$
\begin{array}{cccc}
\vdots & \vdots & \vdots & \vdots \\
\forall\left(o_{1}, \forall\left(p^{\prime}, t\right)\right) & \forall\left(o_{2}, \forall\left(q^{\prime}, \bar{t}\right)\right) & \forall\left(o_{3}, p^{\prime}\right) & \forall\left(o_{4}, q^{\prime}\right) \\
\cline { 1 - 2 } & \forall(p, \bar{q}) &
\end{array}
$$

(b) If $u=\{p, q\}^{\Gamma}$ and $v=\left\{o^{\prime}\right\}^{\Gamma}$, where $o^{\prime} \in O_{\{\exists(p, q)\}, \Gamma}$, then, by Lemmas 3.1 and 3.2, there exist $o_{1}, o_{2}, o_{3} \in\{p, q\}$ such that $\Gamma \vdash$ $\forall\left(o_{1}, \forall\left(p^{\prime}, t\right)\right), \Gamma \vdash \forall\left(o_{2}, \forall\left(q^{\prime}, \bar{t}\right)\right), \Gamma \vdash \exists\left(o_{3}, o^{\prime}\right), \Gamma \vdash \forall\left(o^{\prime}, p^{\prime}\right)$ and $\Gamma \vdash$ $\forall\left(o^{\prime}, q^{\prime}\right)$ Thus we have the derivation

$$
\begin{array}{ccccc}
\vdots & \vdots & \vdots & \vdots & \vdots \\
\forall\left(o_{1}, \forall\left(p^{\prime}, t\right)\right) & \forall\left(o_{2},, \forall\left(q^{\prime}, \bar{t}\right)\right) & \exists\left(o_{3}, o^{\prime}\right) & \forall\left(o^{\prime}, p^{\prime}\right) & \forall\left(o^{\prime}, q^{\prime}\right) \\
\text { (G2). }
\end{array}
$$

(c) If $v=\{p, q\}^{\Gamma}$ and $u=\{o\}^{\Gamma}$, where $o \in O_{\{\exists(p, q)\}, \Gamma \text {, then, by Lem- }}$ mas 3.1 and 3.2, there exist $o_{1}, o_{2}, o_{3} \in\{p, q\}$ such that $\Gamma \vdash \forall\left(o_{1}, o\right)$, $\Gamma \vdash \forall\left(o, \forall\left(p^{\prime}, t\right)\right), \Gamma \vdash \forall\left(o, \forall\left(q^{\prime}, \bar{t}\right)\right), \Gamma \vdash \forall\left(o_{2}, p^{\prime}\right)$ and $\Gamma \vdash \forall\left(o_{3}, q^{\prime}\right)$. Thus we have the derivation

$$
\begin{array}{ccccc}
\vdots & \vdots & \vdots & \vdots & \vdots \\
\exists\left(o_{1}, o\right) & \forall\left(o, \forall\left(p^{\prime}, t\right)\right) & \forall\left(o, \forall\left(q^{\prime}, \bar{t}\right)\right) & \forall\left(o_{2}, p^{\prime}\right) & \forall\left(o_{3}, q^{\prime}\right) \\
\end{array}
$$


(d) If $u=\{o\}^{\Gamma}$ and $v=\left\{o^{\prime}\right\}^{\Gamma}$, where $o, o^{\prime} \in O_{\{\exists(p, q)\}, \Gamma}$, then by Lemma 3.1, we have $\Gamma \vdash \forall\left(o, \forall\left(p^{\prime}, r\right)\right), \Gamma \vdash \forall\left(o, \forall\left(q^{\prime}, \bar{r}\right)\right), \Gamma \vdash \forall\left(o^{\prime}, p^{\prime}\right)$, and $\Gamma \vdash \forall\left(o^{\prime}, q^{\prime}\right)$; and by Lemma 3.1, exchanging $p$ and $q$ if necessary, we have $\Gamma \vdash \exists\left((p, o)\right.$, and either $\Gamma \vdash \exists\left(\left(p, o^{\prime}\right)\right.$ or $\Gamma \vdash \exists\left(q, o^{\prime}\right)$. Thus we have either the derivation

$$
\begin{array}{cccccc}
\vdots & \vdots & \vdots & \vdots & \vdots & \vdots \\
\exists((p, o) & \exists y\left(q, o^{\prime}\right) & \forall\left(o, \forall\left(p^{\prime}, t\right)\right) & \forall\left(o, \forall\left(q^{\prime}, \bar{t}\right)\right) & \forall\left(o^{\prime}, p^{\prime}\right) & \forall\left(o^{\prime}, q^{\prime}\right) \\
\hline \forall(p, \bar{q}) &
\end{array}
$$

or the derivation

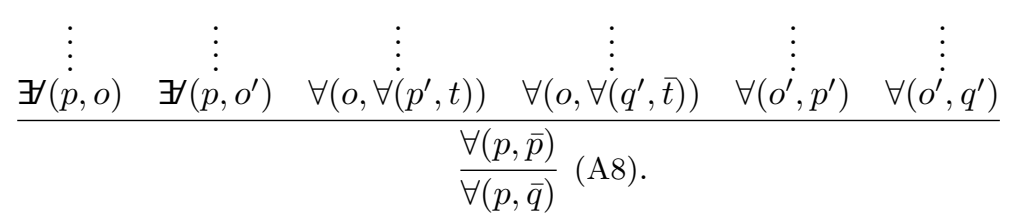

Lemma 3.15. Suppose $\Phi \models \psi$, where $\psi$ is of any of the forms $\forall(p, q)$, $\forall(p, \exists(q, t)), \exists(q, \forall(p, t))$ or $\exists(p, q)$. Then $\Phi \vdash \psi$.

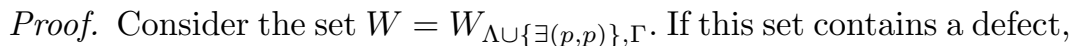
then $\Phi \vdash \forall(p, \bar{p})$ by Lemmas 3.13 and 3.14. Further, if $\psi=\exists(q, \forall(p, t))$, then since $\Phi \models \exists(q, q)$, Lemma 3.12 guarantees $\Phi \vdash \exists(q, q)$. Hence we have one of the derivations:

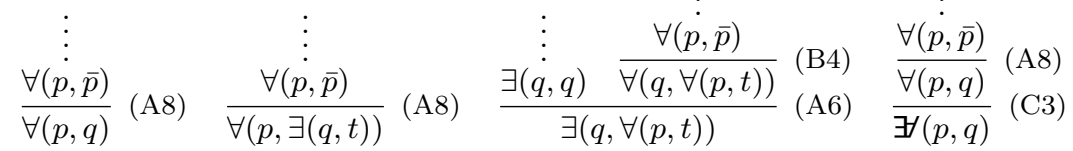

as required. Therefore, we may assume that $W$ contains no defect. Let $\mathfrak{A}=\mathfrak{A}_{\Lambda \cup\{\exists(p, p)\}, \Gamma}$ and $\overline{\mathfrak{A}}=\overline{\mathfrak{A}}_{\Lambda \cup\{\exists(p, p)\}, \Gamma}$. By Lemma 3.6, both $\mathfrak{A}$ and $\overline{\mathfrak{A}}$ are models of $\Phi$, and hence of $\psi$. We consider the various forms of $\psi$ in turn.

$\psi=\forall(p, q)$ : By construction of $\mathfrak{A}, a=\left(\{p\}^{\Gamma}, 1\right) \in p^{\mathfrak{A}}$. Since $\mathfrak{A} \models \psi$, $a \in q^{\mathfrak{A}}$, whence $q \in\{p\}^{\Gamma}$, whence $\Phi \vdash \forall(p, q)$, by Lemma 3.2.

$\psi=\forall(p, \exists(q, t))$ : We assume first that $t$ is positive, and write $t=r$. By construction of $\mathfrak{A}, a=\left(\{p\}^{\Gamma}, 1\right) \in p^{\mathfrak{A}}$. Since $\mathfrak{A} \models \psi$, there exists $b \in q^{\mathfrak{A}}$ such that $\langle a, b\rangle \in r^{\mathfrak{A}}$. Thus, there exists $q^{\prime}$ such that either (i) $b=\left(\left\{q^{\prime}\right\}^{\Gamma}, 1\right)$ with $\exists\left(q^{\prime}, r\right) \in\{p\}^{\Gamma}$ and $q \in\left\{q^{\prime}\right\}^{\Gamma}$, or (ii) $b=(v, j)$ with $\forall\left(q^{\prime}, r\right) \in\{p\}^{\Gamma}$ and $q, q^{\prime} \in v$. In case (i), by Lemma 3.2, $\Phi \vdash \forall\left(p, \exists\left(q^{\prime}, r\right)\right)$ 
and $\Phi \vdash \forall\left(q^{\prime}, q\right)$, whence we have the derivation

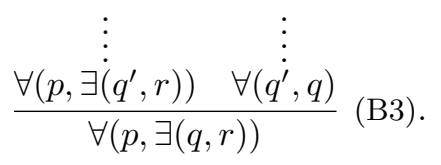

In case (ii), by Lemma 3.2, $\Phi \vdash \forall\left(p, \forall\left(q^{\prime}, r\right)\right)$; moreover, either (a) $v \in W_{\Lambda, \Gamma}$, in which case $\Phi \vdash \exists\left(q^{\prime}, q\right)$, by Lemma 3.9 (ii), or (b) $v=$ $\{o\}^{\Gamma}$, where $o \in O_{\{\exists(p, p)\}, \Gamma}$, in which case, by Lemmas 3.1 and 3.2, $\Phi \vdash \exists(p, o), \Phi \vdash \forall(o, q)$, and $\Phi \vdash \forall\left(o, q^{\prime}\right)$. Hence, we have either of the derivations

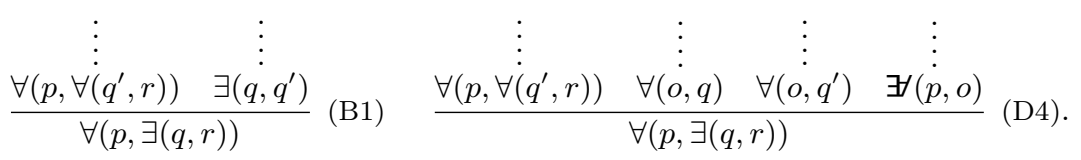

If $t=\bar{r}$ is negative, then we proceed as before, but replacing $\mathfrak{A}$ by $\overline{\mathfrak{A}}$.

$\psi=\exists(q, \forall(p, t))$ : Assume first that $t=r$ is positive, and recall our earlier observation that $\Phi \vdash \exists(q, q)$. By construction, $b=\left(\{p\}^{\Gamma}, 0\right) \in$ $p^{\mathfrak{A}}$. Since $\mathfrak{A} \models \psi$, let $a=(u, i)$ be such that $a \in q^{\mathfrak{A}}$ and $\langle a, b\rangle \in r^{\mathfrak{A}}$. Thus there exists $p^{\prime}$ such that $\forall\left(p^{\prime}, r\right) \in u$ and $p^{\prime} \in\{p\}^{\Gamma}$. Now, either:

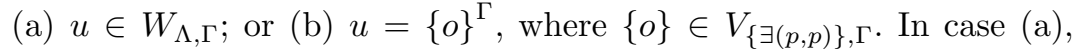
$\Phi \vdash \exists\left(q, \forall\left(p^{\prime}, r\right)\right)$ by Lemma 3.9 (ii), and $\Phi \vdash \forall\left(p, p^{\prime}\right)$ by Lemma 3.2, whence we have the derivation

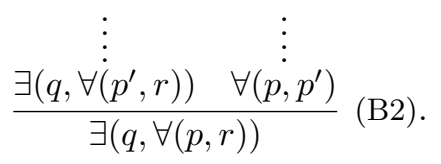

In case (b), by Lemmas 3.1 and 3.2, $\Phi \vdash \exists(p, o), \Phi \vdash \forall(o, q), \Phi \vdash$ $\forall\left(o, \forall\left(p^{\prime}, r\right)\right)$ and $\Phi \vdash \forall\left(p, p^{\prime}\right)$, whence we have the derivation

$$
\begin{array}{ccccc} 
& & \vdots & \vdots & \\
\vdots & \vdots & \forall\left(o, \forall\left(p^{\prime}, r\right)\right) & \forall\left(p, p^{\prime}\right) & \vdots \\
\exists(q, q) & \exists((p, o) & (\mathrm{B} 2) \quad \forall(o, q) & (\mathrm{D} 2) .
\end{array}
$$

If $t=\bar{r}$ is negative, then we proceed as before, but replacing $\mathfrak{A}$ by $\overline{\mathfrak{A}}$.

$\psi=\mathfrak{\exists}(p, q)$ : By construction, $a=\left(\{p\}^{\Gamma}, 1\right) \in p^{\mathfrak{A}}$. Since $\mathfrak{A}=\psi$, let $b=(v, j)$ be such that $b \in q^{\mathfrak{A}}$. Thus, $q \in v$. Either (a) $v \in W_{\Lambda, \Gamma}$ or (b)

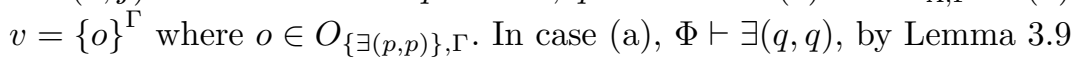


(ii), and we have the derivation

$$
\begin{gathered}
\vdots \\
\frac{\exists(q, q)}{\exists(p, q)}(\mathrm{C} 5) .
\end{gathered}
$$

In case (b), $\Phi \vdash \exists(p, q)$ by Lemma 3.1, and we are done.

Lemma 3.16. Suppose $\Phi \models \forall(p, \bar{q})$. Then $\Phi \vdash \forall(p, \bar{q})$.

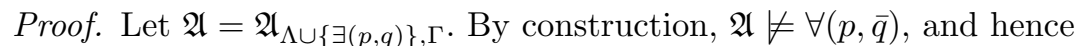
$\mathfrak{A} \not \models \Phi$. By Lemma 3.6, $W_{\Lambda \cup\{\exists(p, q)\}, \Gamma}$ contains a defect. By Lemmas 3.13 and $3.14, \Phi \vdash \forall(p, \bar{q})$.

Lemma 3.17. If $W_{\Lambda \cup\{\exists(p, p), \exists(q, q)\}, \Gamma}$ contains a defect, then, for any binary literal $t, \Phi \vdash \forall(p, \forall(q, t))$.

Proof. If $W_{\Lambda \cup\{\exists(p, p)\}, \Gamma}$ contains a defect, then, by Lemmas 3.13 and 3.14 (setting $q=p), \Phi \vdash \forall(p, \bar{p})$. Similarly, if $W_{\Lambda \cup\{\exists(q, q)\}, \Gamma}$ contains a defect, then $\Phi \vdash \forall(q, \bar{q})$. Hence we have either of the derivations

$$
\begin{array}{cc}
\vdots & \vdots \\
\frac{\forall(p, \bar{p})}{\forall(p, \forall(q, t))}(\mathrm{A} 8) & \frac{\forall(\bar{q}, \bar{q})}{\forall(p, \forall(q, t))}(\mathrm{B} 4) .
\end{array}
$$

The only other possibility is a global defect involving one element of $W_{\{\exists(p, p)\}, \Gamma}$ and another of $W_{\{\exists(q, q)\}, \Gamma}$. Suppose, then, $\forall\left(p^{\prime}, t^{\prime}\right), \forall\left(q^{\prime}, \bar{t}^{\prime}\right) \in$

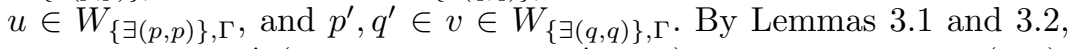
there exist $o, o^{\prime}$ (possibly, $o=p, o^{\prime}=q$ ) such that $\Phi \vdash \exists(p, o)$, $\Phi \vdash \forall\left(o, \forall\left(p^{\prime}, t^{\prime}\right)\right), \Phi \vdash \forall\left(o, \forall\left(q^{\prime}, \bar{t}^{\prime}\right)\right), \Phi \vdash \exists\left(q, o^{\prime}\right), \Phi \vdash \forall\left(o^{\prime}, p^{\prime}\right)$ and $\Phi \vdash \forall\left(o^{\prime}, q^{\prime}\right)$. Thus, we have the derivation

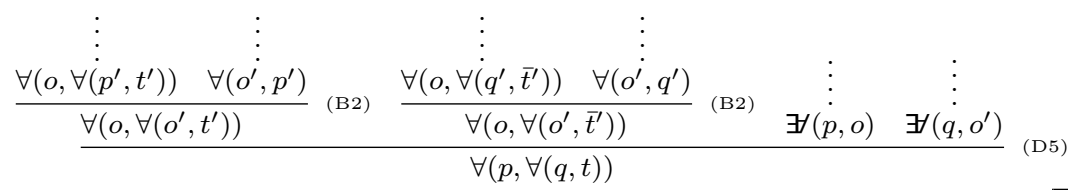

Lemma 3.18. Suppose $\Phi \models \forall(p, \forall(q, t))$. Then $\Phi \vdash \forall(p, \forall(q, t))$.

Proof. Assume for the moment $t$ is positive and write $t=r$. Let $\mathfrak{A}=\mathfrak{A}_{\Lambda \cup\{\exists(p, p), \exists(q, q)\}, \Gamma}$. If $W_{\Lambda \cup\{\exists(p, p), \exists(q, q)\}, \Gamma}$ contains a defect, then the conclusion follows by Lemma 3.17. Otherwise, by Lemma 3.6, $\mathfrak{A} \models$ $\Phi$, whence $\mathfrak{A} \models \forall(p, \forall(q, t))$, and, by construction, the domain of $\mathfrak{A}$ contains $a=\left(\{p\}^{\Gamma}, 1\right)$ and $b=\left(\{q\}^{\Gamma}, 0\right)$. Since $a \in p^{\mathfrak{A}}$ and $b \in q^{\mathfrak{A}}$, we have $\langle a, b\rangle \in r^{\mathfrak{A}}$, whence $\forall\left(q^{\prime}, r\right) \in\{p\}^{\Gamma}$ for some $q^{\prime} \in\{q\}^{\Gamma}$. By 
Lemma 3.2, $\Gamma \vdash \forall\left(p, \forall\left(q^{\prime}, r\right)\right)$ and $\Gamma \vdash \forall\left(q, q^{\prime}\right)$, whence we have the derivation:

$$
\frac{\forall\left(p, \forall\left(q^{\prime}, r\right)\right)}{\forall\left(p, \forall\left(q, q^{\prime}\right)\right.} \text { (B2). }
$$

If $t$ is negative, we write $t=\bar{r}$ and proceed as above, but replacing $\mathfrak{A}$ by $\overline{\mathfrak{A}}=\overline{\mathfrak{A}}_{\Lambda \cup\{\exists(p, p), \exists(q, q)\}, \Gamma}$.

We turn finally to conclusions of the form $\exists(p, \bar{q})$. Fixing the unary

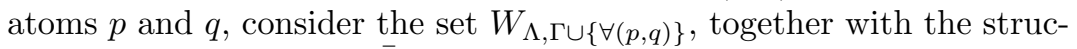
tures $\mathfrak{A}_{\Lambda, \Gamma \cup\{\forall(p, q)\}}$ and $\overline{\mathfrak{A}}_{\Lambda, \Gamma \cup\{\forall(p, q)\}}$, as defined in Sec. 3.2. By way of preparing the ground, let us examine $W_{\Lambda, \Gamma \cup\{\forall(p, q)\}}$ and its relation to $W_{\Lambda, \Gamma}$. Evidently, if $p \notin \bigcup W_{\Lambda, \Gamma}$, then $W_{\Lambda, \Gamma \cup\{\forall(p, q)\}}=W_{\Lambda, \Gamma}$. So

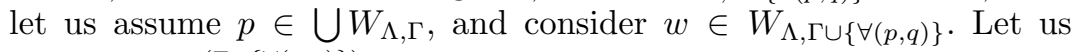
write $w=s^{(\Gamma \cup\{\forall(p, q)\})}$, where $s \in V_{\Lambda, \Gamma \cup\{\forall(p, q)\}}$. Observe that, since $p \in \bigcup W_{\Lambda, \Gamma}$, we in fact have $V_{\Lambda, \Gamma \cup\{\forall(p, q)\}}=V_{\Lambda, \Gamma} \cup V_{\{\exists(q, q)\}, \Gamma \text {. We }}$ may therefore distinguish two cases: either $s \in V_{\Lambda, \Gamma}$ or $V_{\{\exists(q, q)\}, \Gamma}$. But note that, in either case, if $p \notin s^{\Gamma}$, then $s^{(\Gamma \cup\{\forall(p, q)\})}=s^{\Gamma}$; otherwise $s^{(\Gamma \cup\{\forall(p, q)\})}=s^{\Gamma} \cup\{q\}^{\Gamma}$. Fig. 1 illustrates the four possible kinds of elements of $W_{\Lambda, \Gamma \cup\{\forall(p, q)\}}$ that result: elements of the form $s^{\Gamma}$, where $s \in V_{\Lambda, \Gamma}$, but $p \notin s^{\Gamma}$; elements of the form $s^{\Gamma} \cup\{q\}^{\Gamma}$, where $s \in V_{\Lambda, \Gamma}$, and $p \in s^{\Gamma}$; elements of the form $\{o\}^{\Gamma}$, where $\{o\} \in V_{\{\exists(q, q)\}, \Gamma \text {, but }}$ $p \notin\{o\}^{\Gamma}$; elements of the form $\{o\}^{\Gamma} \cup\{q\}^{\Gamma}$, where $\{o\} \in V_{\{\exists(q, q)\}, \Gamma}$, and $p \in\{o\}^{\Gamma}$. Indeed, if $c, d$ are c-terms realized in the same element $w$ of $W_{\Lambda, \Gamma \cup\{\forall(p, q)\}}$, one of the following six cases obtains. To aid readability, we write $[q]^{\Gamma}$ for the set of unary atoms $\{o \mid q \stackrel{\Pi}{\rightarrow} o\}=O_{\{\exists(q, q)\}, \Gamma}$.

(i) there exists $w^{\prime} \in W_{\Lambda, \Gamma}$ such that $c, d \in w^{\prime}$;

(ii) there exists $w^{\prime} \in W_{\Lambda, \Gamma}$ such that $c, p \in w^{\prime}$, and $d \in\{q\}^{\Gamma}$;

(iii) there exists $w^{\prime} \in W_{\Lambda, \Gamma}$ such that $d, p \in w^{\prime}$, and $c \in\{q\}^{\Gamma}$;

(iv) $p \in \bigcup W_{\Lambda, \Gamma}$, and there exists $o \in[q]^{\Gamma}$ such that $c, d \in\{o\}^{\Gamma}$;

(v) $p \in \bigcup W_{\Lambda, \Gamma}$, and there exists $o \in[q]^{\Gamma}$ such that $c, p \in\{o\}^{\Gamma}$ and $d \in\{q\}^{\Gamma}$

(vi) $p \in \bigcup W_{\Lambda, \Gamma}$, and there exists $o \in[q]^{\Gamma}$ such that $d, p \in\{o\}^{\Gamma}$ and $c \in\{q\}^{\Gamma}$.

This six-fold division of cases will be used extensively in the next two lemmas.

Lemma 3.19. If $W_{\Lambda, \Gamma \cup\{\forall(p, q)\}}$ contains a local defect, then $\Phi \vdash \exists(p, \bar{q})$. 

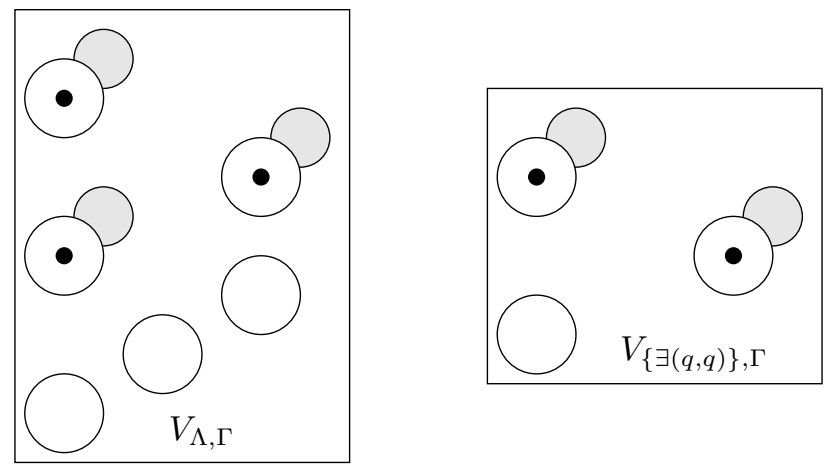

FIGURE 1: Schematic illustration of $W_{\Lambda, \Gamma \cup\{\forall(p, q)\}}$ when $p \in \bigcup W_{\Lambda, \Gamma}$ : white circles illustrate the elements $s^{\Gamma}$, where $s$ is in either $V_{\Lambda, \Gamma}$ or $V_{\{\exists(q, q)\}, \Gamma}$, as indicated by the labels in the rectangles; black dots indicate the presence of the unary atom $p$ in these sets; grey blobs indicate the sets of added elements $\{q\}^{\Gamma}$.

Proof. We have six cases to consider. (i) If $c, \bar{c} \in w \in W_{\Lambda, \Gamma}, \Phi$ is inconsistent, by Lemma 3.10, and the result follows by rule (A7). (ii) If $c, p \in w \in W_{\Lambda, \Gamma}$, and $\bar{c} \in\{q\}^{\Gamma}$, then by Lemma 3.9 (ii), $\Phi \vdash \exists(p, c)$ and by Lemma 3.2, $\Phi \vdash \forall(q, \bar{c})$; hence we have the derivation

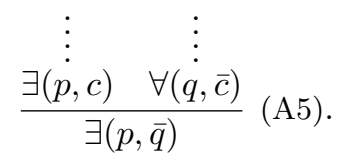

(iii) If $\bar{c}, p \in w \in W_{\Lambda, \Gamma}$, and $c \in\{q\}^{\Gamma}$, we replace $c$ by $\bar{c}$ and proceed as in case (ii). (iv) If $p \in \bigcup W_{\Lambda, \Gamma}, o \in[q]^{\Gamma}$ and $c, \bar{c} \in\{o\}^{\Gamma}$, then, by Lemma 3.9 (ii), $\Phi \vdash \exists(p, p)$, and by Lemmas 3.1 and $3.2, \Gamma \vdash \exists(q, o)$, $\Gamma \vdash \forall(o, c)$ and $\Gamma \vdash \forall(o, \bar{c})$; hence we have the derivation

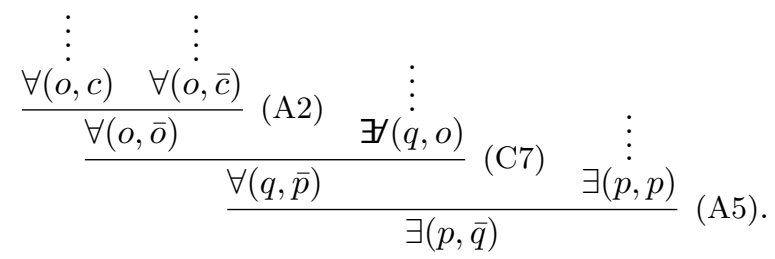

(v) If $p \in \bigcup W_{\Lambda, \Gamma}, o \in[q]^{\Gamma} c, p \in\{o\}^{\Gamma}$ and $\bar{c} \in\{q\}^{\Gamma}$, then, by Lemma 3.9 (ii), $\Phi \vdash \exists(p, p)$, and by Lemmas 3.1 and 3.2, $\Gamma \vdash \exists(q, o)$, 
222 / IAn Pratt-Hartmann

$\Gamma \vdash \forall(o, c), \Gamma \vdash \forall(o, p)$ and $\Gamma \vdash \forall(q, \bar{c})$; hence we have the derivation

$$
\begin{array}{ccccc} 
& & & \vdots & \vdots \\
\vdots & \vdots & \vdots & \forall(o, c) & \forall(\bar{q}, \bar{c}) \\
\exists(p, p) & \exists((\mathrm{q}, o) & \forall(o, p) & \frac{\forall(o, \bar{q})}{(\mathrm{A} 2)} \text { (D3) } \\
\hline & \exists(p, \bar{q}) & &
\end{array}
$$

(vi) If $p \in \bigcup W_{\Lambda, \Gamma}, o \in[q]^{\Gamma} \bar{c}, p \in\{o\}^{\Gamma}$ and $c \in\{q\}^{\Gamma}$, we replace $c$ by $\bar{c}$ and proceed as in case $(\mathrm{v})$.

Lemma 3.20. If $W_{\Lambda, \Gamma \cup\{\forall(p, q)\}}$ contains a global defect, then $\Phi \vdash$ $\exists(p, \bar{q})$.

Proof. Let $u, v$ be elements of $W_{\Lambda, \Gamma \cup\{\forall(p, q)\}}$ and $t$ a binary literal such that $\forall\left(p^{\prime}, t\right), \forall\left(q^{\prime}, \bar{t}\right) \in u$, and $p^{\prime}, q^{\prime} \in v$. We apply the six-fold distinction noted above to both $u$ and $v$, yielding thirty-six cases in total.

(i) Suppose $\forall\left(p^{\prime}, t\right), \forall\left(q^{\prime}, \bar{t}\right) \in u^{\prime} \in W_{\Lambda, \Gamma}$. By Lemma 3.9 (i), there exist $o, Q$ such that $\Phi \vdash Q\left(o, \forall\left(p^{\prime}, t\right)\right)$ and $\Phi \vdash \bar{Q}\left(o, \forall\left(q^{\prime}, \bar{t}\right)\right)$. Hence we have the derivation

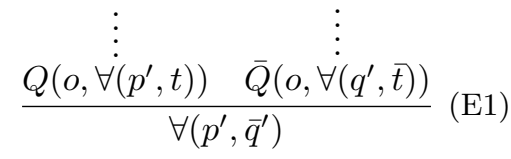

Recall that $\forall\left(p^{\prime}, \bar{q}^{\prime}\right)$ is symmetric in $p^{\prime}$ and $q^{\prime}$. We consider the subcases: (a) $p^{\prime}, q^{\prime}, \in v^{\prime} \in W_{\Lambda, \Gamma}$; (b) $p^{\prime}, p \in v^{\prime} \in W_{\Lambda, \Gamma}$, and $q^{\prime} \in\{q\}^{\Gamma}$; (c) $q^{\prime}, p \in v^{\prime} \in W_{\Lambda, \Gamma}$, and $p^{\prime} \in\{q\}^{\Gamma} ;(\mathrm{d}) p^{\prime}, q^{\prime} \in\{o\}^{\Gamma}$, where $o \in[q]^{\Gamma}$, and $p \in \bigcup W_{\Lambda, \Gamma} ;$ (e) $p^{\prime}, p \in\{o\}^{\Gamma}$, where $o \in[q]^{\Gamma}, q^{\prime} \in\{q\}^{\Gamma}$, and $p \in \bigcup W_{\Lambda, \Gamma}$; (f) $q^{\prime}, p \in\{o\}^{\Gamma}$, where $o \in[q]^{\Gamma}, p^{\prime} \in\{q\}^{\Gamma}$, and $p \in \bigcup W_{\Lambda, \Gamma}$. If (a), by Lemma 3.9 (ii), $\Phi \vdash \exists\left(p^{\prime}, q^{\prime}\right)$, and we have the derivation

$$
\frac{\forall\left(p^{\prime}, \bar{q}^{\prime}\right) \quad \exists\left(p^{i}, q^{\prime}\right)}{\frac{\exists\left(p^{\prime}, \bar{p}^{\prime}\right)}{\exists(p, \bar{q})}(\mathrm{A} 7) .}
$$

If (b), by Lemma 3.9 (ii), $\Phi \vdash \exists\left(p^{\prime}, p\right)$, and by Lemma 3.2, $\Phi \vdash \forall\left(q, q^{\prime}\right)$. Hence, we have the derivation

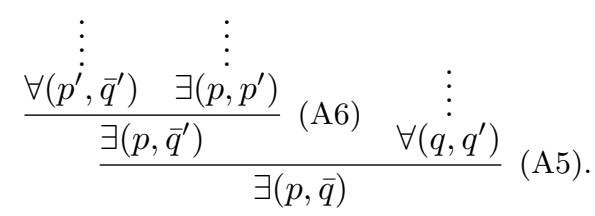


Sub-case (c) is identical to (b) by transposing $p^{\prime}$ and $q^{\prime}$. If (d), by Lemmas 3.1 and 3.2, $\Gamma \vdash \exists(q, o), \Gamma \vdash \forall\left(o, p^{\prime}\right)$, and $\Gamma \vdash \forall\left(o, q^{\prime}\right)$, and by Lemma 3.9 (ii), $\Phi \vdash \exists(p, p)$. Hence, we have the derivation:

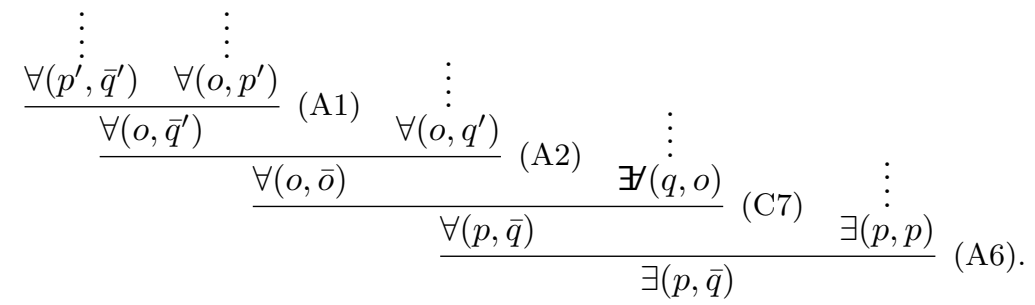

If (e), then by Lemmas 3.1 and 3.2, $\Gamma \vdash \exists(q, o), \Gamma \vdash \forall\left(o, p^{\prime}\right), \Gamma \vdash \forall(o, p)$ and $\Gamma \vdash \forall\left(q, q^{\prime}\right)$, and by Lemma 3.9 (ii), $\Phi \vdash \exists(p, p)$. Hence, we have the derivation:

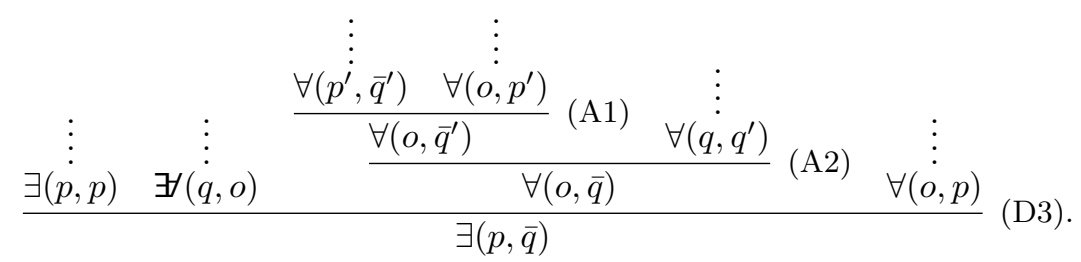

Sub-case (f) is identical to (e) by transposing $p^{\prime}$ and $q^{\prime}$.

(ii) Suppose $\forall\left(p^{\prime}, t\right), p \in u^{\prime} \in W_{\Lambda, \Gamma}$, and $\forall\left(q^{\prime}, \bar{t}\right) \in\{q\}^{\Gamma}$. By Lemma 3.9 (ii), $\Phi \vdash \exists\left(p, \forall\left(p^{\prime}, t\right)\right)$, and by Lemma $3.2, \Phi \vdash \forall\left(q, \forall\left(q^{\prime}, \bar{t}\right)\right)$. We consider the sub-cases (a)-(f) regarding $v$, exactly as in Case (i). If (a), by Lemma 3.9 (ii), $\Phi \vdash \exists\left(p^{\prime}, q^{\prime}\right)$, whence we have the derivation

$$
\frac{\exists\left(p, \forall\left(p^{\prime}, t\right)\right) \quad \exists\left(p^{\prime}, q^{\prime}\right)}{\frac{\exists\left(p, \exists\left(q^{\prime}, t\right)\right)}{3}(\mathrm{~B} 1) \quad \forall\left(q, \forall\left(q^{\prime}, \bar{t}\right)\right)} \text { (A5). }
$$

If (b), by Lemma 3.9 (ii), $\Phi \vdash \exists\left(p, p^{\prime}\right)$, and by Lemma 3.2, $\Phi \vdash \forall\left(q, q^{\prime}\right)$, whence we have the derivation

$$
\begin{array}{cccc}
\vdots & \vdots & \vdots & \vdots \\
\frac{\exists\left(p, \forall\left(p^{\prime}, t\right)\right)}{\exists\left(p, p^{\prime}\right)} & (\mathrm{B} 1) & \frac{\forall\left(q, \forall\left(q^{\prime}, \bar{t}\right)\right)}{\forall\left(q, \forall\left(q, q^{\prime}\right)\right.} & \text { (B2) } \\
\hline & \exists(p, \bar{q}) & \text { (E3). }
\end{array}
$$

If (c), by Lemma 3.9 (ii), $\Phi \vdash \exists\left(p, q^{\prime}\right)$, and by Lemma $3.2, \Phi \vdash \forall\left(q, p^{\prime}\right)$, 
224 / IAn Pratt-Hartmann

whence we have the derivation

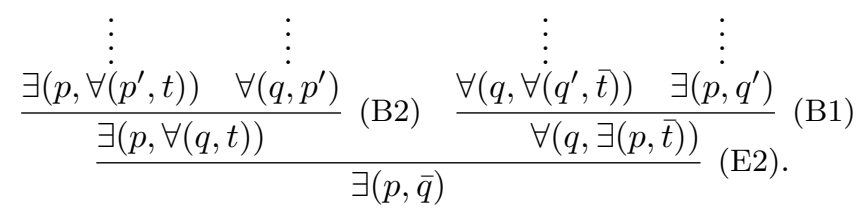

If (d), by Lemmas 3.1 and 3.2, $\Phi \vdash \exists(q, o), \Phi \vdash \forall\left(o, p^{\prime}\right)$, and $\Phi \vdash$ $\forall\left(o, q^{\prime}\right)$, whence we have the derivation

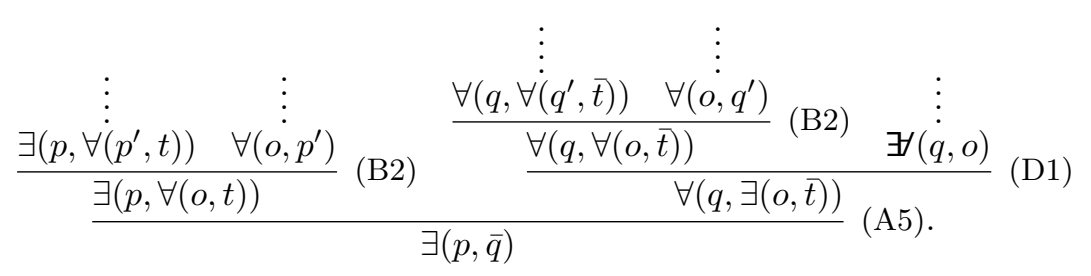

If (e), then by Lemmas 3.1 and 3.2, $\Gamma \vdash \exists\left((q, o), \Gamma \vdash \forall\left(o, p^{\prime}\right), \Gamma \vdash \forall(o, p)\right.$ and $\Gamma \vdash \forall\left(q, q^{\prime}\right)$. Hence, we have the derivation:

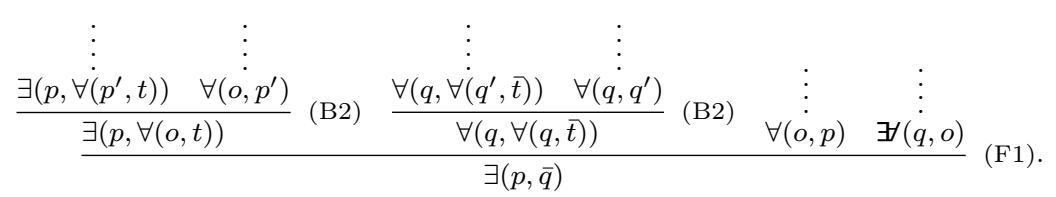

If (f), then by Lemmas 3.1 and 3.2, $\Gamma \vdash \exists(q, o), \Gamma \vdash \forall\left(o, q^{\prime}\right), \Gamma \vdash \forall(o, p)$ and $\Gamma \vdash \forall\left(q, p^{\prime}\right)$. Hence, we have

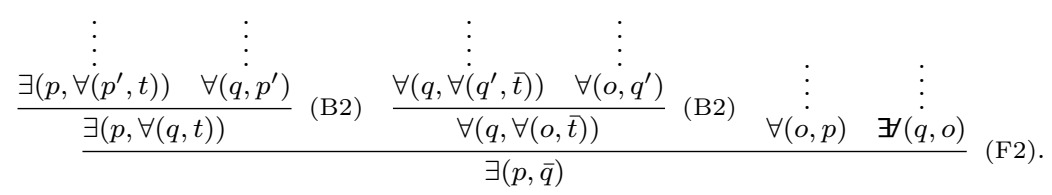

(iii) Suppose $\forall\left(q^{\prime}, \bar{t}\right), p \in u^{\prime} \in W_{\Lambda, \Gamma}$, and $\forall\left(p^{\prime}, t\right) \in\{q\}^{\Gamma}$. We argue exactly as for case (ii), with $p^{\prime}$ and $q^{\prime}$ exchanged, and $t$ replaced by $\bar{t}$.

(iv) Suppose $\forall\left(p^{\prime}, t\right), \forall\left(q^{\prime}, \bar{t}\right) \in\left\{o^{\prime}\right\}^{\Gamma}$, where $o^{\prime} \in[q]^{\Gamma}$; and suppose $p \in$ $\bigcup W_{\Lambda, \Gamma}$. By Lemmas 3.1 and 3.2, $\Phi \vdash \exists\left(q, o^{\prime}\right), \Phi \vdash \forall\left(o^{\prime}, \forall\left(p^{\prime}, t\right)\right)$, and $\Phi \vdash \forall\left(o^{\prime}, \forall\left(q^{\prime}, \bar{t}\right)\right)$; and by Lemma 3.9 (ii), $\Phi \vdash \exists(p, p)$. We consider the sub-cases (a)-(f) regarding $v$ exactly as in Case (i). If (a), by Lemma 3.9 
(ii), $\Phi \vdash \exists\left(p^{\prime}, q^{\prime}\right)$, whence we have the derivation

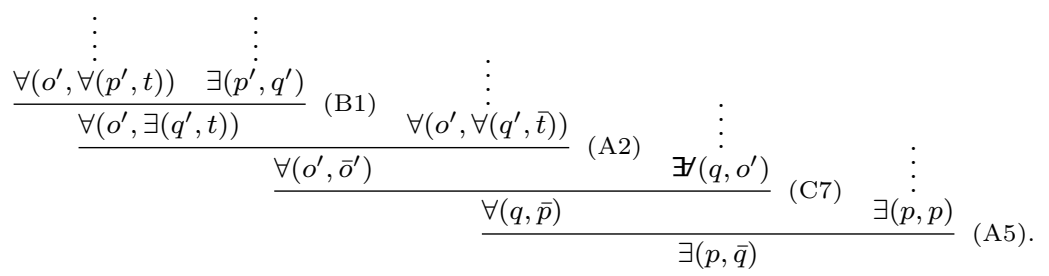

If (b), by Lemma 3.9 (ii), $\Phi \vdash \exists\left(p, p^{\prime}\right)$, and by Lemma 3.2, $\Phi \vdash \forall\left(q, q^{\prime}\right)$, whence we have the derivation

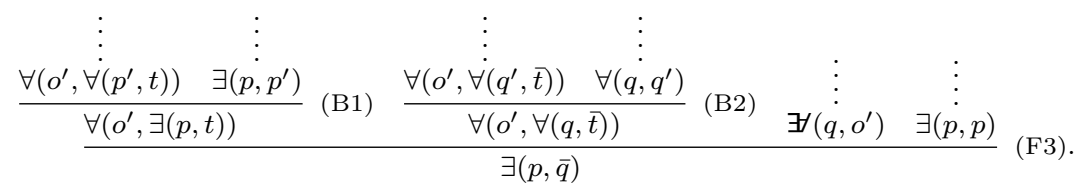

Sub-case (c) is identical to (b) by transposing $p^{\prime}$ and $q^{\prime}$ and replacing $t$ by $\bar{t}$. If (d), by Lemmas 3.1 and 3.2, $\Phi \vdash \exists(q, o) \Phi \vdash \forall\left(o, p^{\prime}\right)$ and $\Phi \vdash \forall\left(o, q^{\prime}\right)$, whence we have the derivations

$$
\begin{array}{cccc}
\vdots & \vdots & \vdots & \vdots \\
\forall\left(o^{\prime}, \forall\left(p^{\prime}, t\right)\right) & \forall\left(o, p^{\prime}\right) & (\mathrm{B} 2) & \frac{\forall\left(o^{\prime}, \forall\left(q^{\prime}, \bar{t}\right)\right)}{\forall\left(o^{\prime}, \forall(o, t)\right)}
\end{array}
$$

and hence the derivation

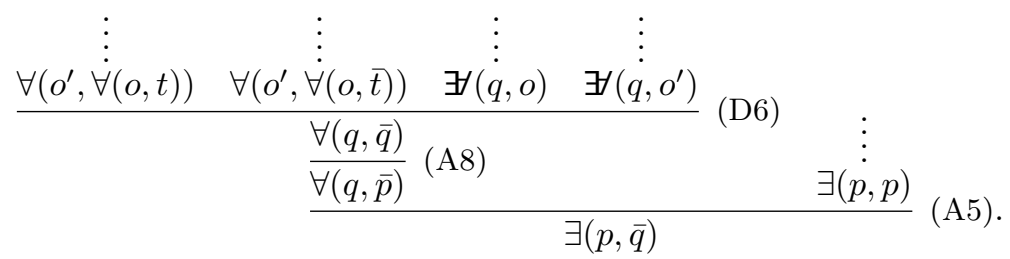

If (e), then by Lemmas 3.1 and 3.2, $\Gamma \vdash \exists(q, o), \Gamma \vdash \forall\left(o, p^{\prime}\right), \Gamma \vdash \forall(o, p)$ and $\Gamma \vdash \forall\left(q, q^{\prime}\right)$, and by Lemma 3.9 (ii), $\Phi \vdash \exists(p, p)$. Hence, we have the derivations:

$$
\begin{array}{cccc}
\vdots & \vdots & \vdots & \vdots \\
\frac{\forall\left(o, \forall\left(p^{\prime}, t\right)\right)}{\forall\left(o, \forall\left(o^{\prime}, t\right)\right)} & \forall\left(o^{\prime}, p^{\prime}\right) & (\mathrm{B} 2) & \frac{\forall\left(o, \forall\left(q^{\prime}, \bar{t}\right)\right)}{\forall(o, \forall(q, \bar{t}))}
\end{array}
$$

and hence the derivation

$$
\begin{array}{cccccc}
\vdots & \vdots & \vdots & \vdots & \vdots & \vdots \\
\forall\left(o, \forall\left(o^{\prime}, t\right)\right) & \forall(o, \forall(q, \bar{t})) & \exists((q, o) & \exists\left(\left(\dot{q}, o^{\prime}\right)\right. & \forall\left(o^{\prime}, p\right) & \exists(p, p)
\end{array}
$$

Case (f) is identical to (e) by transposing $p^{\prime}$ and $q^{\prime}$ and replacing $t$ by $\bar{t}$. 
(v) Suppose $\forall\left(p^{\prime}, t\right), p \in\left\{o^{\prime}\right\}^{\Gamma}$, where $o^{\prime} \in[q]^{\Gamma}, \forall\left(q^{\prime}, \bar{t}\right) \in\{q\}^{\Gamma}$; and suppose $p \in \bigcup W_{\Lambda, \Gamma}$. By Lemmas 3.1 and 3.2, $\Phi \vdash \exists\left(q, o^{\prime}\right), \Phi \vdash$ $\forall\left(o^{\prime}, \forall\left(p^{\prime}, t\right)\right), \Phi \vdash \forall\left(o^{\prime}, p\right), \forall\left(q, \forall\left(q^{\prime}, \bar{t}\right)\right)$; and by Lemma 3.9 (ii), $\Phi \vdash$ $\exists(p, p)$. We consider the cases (a)-(f) regarding $v$ exactly as for (i). If (a), by Lemma 3.9 (ii), $\Phi \vdash \exists\left(p^{\prime}, q^{\prime}\right)$, whence we have the derivation

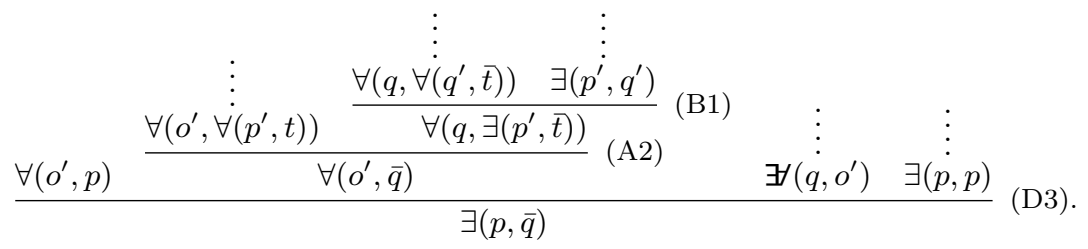

If (b), by Lemma 3.9 (ii), $\Phi \vdash \exists\left(p, p^{\prime}\right)$, and by Lemma 3.2, $\Phi \vdash \forall\left(q, q^{\prime}\right)$, whence we have the derivation

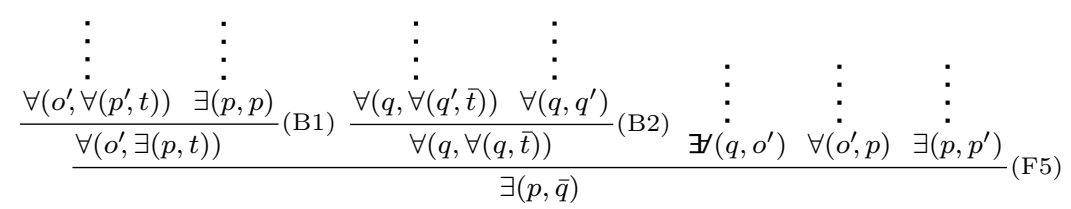

If (c), by Lemma 3.9 (ii), $\Phi \vdash \exists\left(p, q^{\prime}\right)$, and by Lemma $3.2, \Phi \vdash \forall\left(q, p^{\prime}\right)$, whence we have the derivation

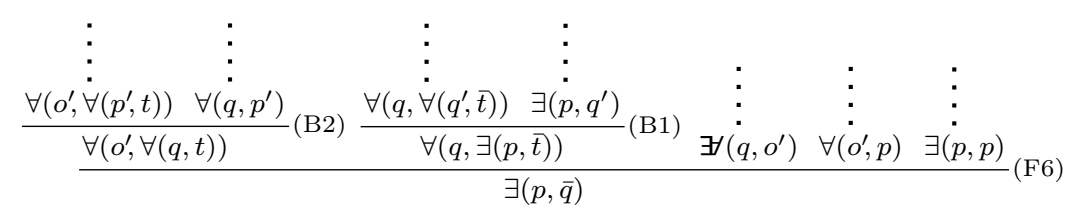

If (d), by Lemmas 3.1 and 3.2, $\Phi \vdash \exists(q, o) \Phi \vdash \forall\left(o, p^{\prime}\right)$ and $\Phi \vdash \forall\left(o, q^{\prime}\right)$, whence we have the derivations

$$
\begin{array}{cccc}
\vdots & \vdots & \vdots & \vdots \\
\frac{\forall\left(o^{\prime}, \forall\left(p^{\prime}, t\right)\right)}{\forall\left(o^{\prime}, \forall(o, t)\right)} & \forall\left(o, p^{\prime}\right) & (\mathrm{B} 2) & \frac{\forall\left(q, \forall\left(q^{\prime}, \bar{t}\right)\right)}{\forall(q, \forall(o, \bar{t}))}
\end{array}
$$

and hence the derivation

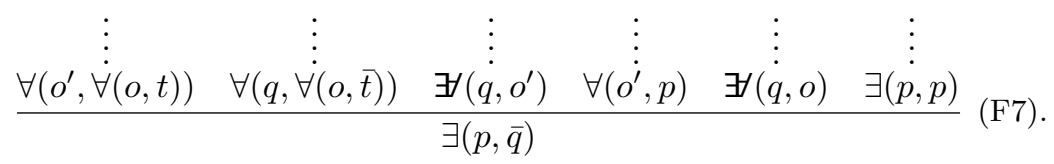

If (e), then by Lemmas 3.1 and 3.2, $\Gamma \vdash \exists(q, o), \Gamma \vdash \forall\left(o, p^{\prime}\right), \Gamma \vdash \forall(o, p)$ and $\Gamma \vdash \forall\left(q, q^{\prime}\right)$, and by Lemma 3.9 (ii), $\Phi \vdash \exists(p, p)$. Hence, we have the derivations:

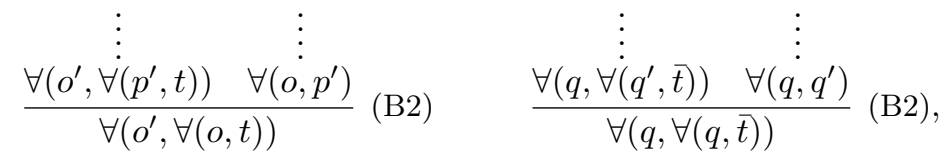


and hence the derivation

\begin{tabular}{ccccccc}
$\vdots$ & $\vdots$ & $\vdots$ & $\vdots$ & $\vdots$ & $\vdots$ & $\vdots$ \\
$\forall\left(o^{\prime}, \forall(o, t)\right)$ & $\forall(q, \forall(q, \bar{t}))$ & $\exists\left(\left(q, o^{\prime}\right)\right.$ & $\forall\left(o^{\prime}, p\right)$ & $\exists(q, o)$ & $\forall(o, p)$ & $\exists(p, p)$ \\
\hline$\exists(p, \bar{q})$ &
\end{tabular}

If (f), then by Lemmas 3.1 and 3.2, $\Gamma \vdash \exists(q, o), \Gamma \vdash \forall\left(o, q^{\prime}\right), \Gamma \vdash \forall(o, p)$ and $\Gamma \vdash \forall\left(q, p^{\prime}\right)$, and by Lemma 3.9 (ii), $\Phi \vdash \exists(p, p)$. Hence, we have the derivations:

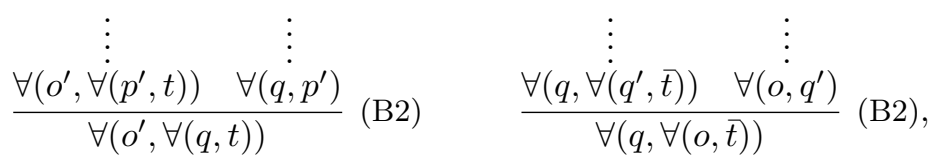

and hence the derivation

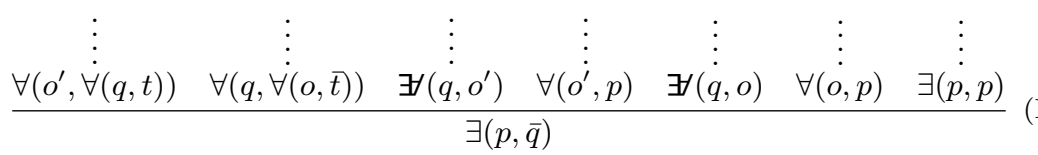

(vi) Suppose $\forall\left(q^{\prime}, \bar{t}\right), p \in\left\{o^{\prime}\right\}^{\Gamma}$, where $o^{\prime} \in[q]^{\Gamma}, \forall\left(p^{\prime}, t\right) \in\{q\}^{\Gamma}$; and suppose $p \in \bigcup W_{\Lambda, \Gamma}$. We argue exactly as for case (v), with $p^{\prime}$ and $q^{\prime}$ exchanged, and $t$ replaced by $\bar{t}$.

Lemma 3.21. Suppose $\Phi \models \exists(p, \bar{q})$. Then $\Phi \vdash \exists(p, \bar{q})$.

Proof. Let $\mathfrak{A}=\mathfrak{A}_{\Lambda, \Gamma \cup\{\forall(p, q)\}}$. By construction, $\mathfrak{A}=\forall(p, q)$, and hence $\mathfrak{A} \not \models \Phi$. By Lemma 3.6, $W_{\Lambda, \Gamma \cup\{\forall(p, q)\}}$ contains a defect. By Lemmas 3.19 and $3.20, \Phi \vdash \exists(p, \bar{q})$.

Proof of Theorem 2.4. Lemmas 3.12, 3.15, 3.16, 3.18 and 3.21.

\section{References}

Corcoran, John. 1972. Completeness of an ancient logic. Journal of Symbolic Logic 37(4):696-702.

Pratt-Hartmann, Ian and Lawrence S. Moss. 2009. Logics for the relational syllogistic. Review of Symbolic Logic 2(4):647-683.

Smiley, T.J. 1973. What is a syllogism? Journal of Philosophical Logic 2:135154. 